\title{
Asymmetric Synthesis of the Dibenzocyclooctadiene Lignans Interiotherin A and Gomisin R
}

\author{
Robert S. Coleman* and Srinivas Reddy Gurrala \\ Department of Chemistry, The Ohio State University, 100 West $18^{\text {th }}$ Avenue, Columbus, Ohio 43210
}

\section{Supporting Information}

General Methods: All ${ }^{1} \mathrm{H}$ and ${ }^{13} \mathrm{C}$ NMR spectra were recorded on a Bruker DRX-500 spectrometer. Chemical shifts are reported in ppm relative to the $\mathrm{CHCl}_{3}$ peak at $7.27 \mathrm{ppm}\left({ }^{1} \mathrm{H}\right)$ or $77.0 \mathrm{ppm}\left({ }^{13} \mathrm{C}\right)$. The Ohio State University Chemistry Mass Spectrometry Facility on a Micromass Q-TOF2 instrument performed all mass spectroscopy. Unless otherwise specified, all reactions were run under an inert atmosphere of nitrogen. All solvents were freshly distilled before use. Yields reported refer to isolated material that was determined to be pure by NMR spectroscopy and thin layer chromatography (TLC), unless specified otherwise in the text.

3-(benzyloxy)-5-bromo-1, 2-diol: Slurry of 3-(benzyloxy)-5-bromo-2-hydroxybenzaldehyde (11) $(8.8 \mathrm{~g}, 28.6 \mathrm{mmol})$ in water $(150 \mathrm{~mL})$ was treated with $3 \mathrm{~N} \mathrm{NaOH}(24 \mathrm{~mL}, 71.6 \mathrm{mmol})$ to obtained pale yellow colored paste. The mixture was diluted with $50 \mathrm{~mL}$ water and treated drop wise with $30 \% \mathrm{H}_{2} \mathrm{O}_{2}(21 \mathrm{~mL}, 186.3 \mathrm{mmol})$ at $25{ }^{\circ} \mathrm{C}$ and was stirred for $3 \mathrm{~h}$ until the yellow colored material becomes free flowing solid. Reaction mixture was diluted with ethyl acetate $(150 \mathrm{~mL})$ and acidified to $\mathrm{pH} 2$, with $6 \mathrm{~N}$ hydrochloric acid and organic layer was separated washed with water and saturated aqueous $\mathrm{NaCl}$. The organic layer was dried $\left(\mathrm{Na}_{2} \mathrm{SO}_{4}\right)$, concentrated, and the residue was purified by flash chromatography (silica, 20\% EtOAc/hexane) to afford pure 3-(benzyloxy)-5-bromo-1, 2-diol (7.01 g, 83\%) as a solid: ${ }^{1} \mathrm{H} \mathrm{NMR}\left(\mathrm{CDCl}_{3}, 500\right.$ MHz) $\delta 7.45-7.35(\mathrm{~m}, 5 \mathrm{H}), 6.80(\mathrm{~d}, 1 \mathrm{H}, J=2.1 \mathrm{~Hz}), 6.71(\mathrm{~d}, 1 \mathrm{H}, J=2.1 \mathrm{~Hz}), 5.39(\mathrm{~s}, 1 \mathrm{H}), 5.37$ (s, 1H), $5.06(\mathrm{~s}, 2 \mathrm{H}) ;{ }^{13} \mathrm{C} \mathrm{NMR}\left(\mathrm{CDCl}_{3}, 125 \mathrm{MHz}\right) \delta 146.6,144.8,135.7,132.0,128.8$ (2 C), 128.7, 128.0 (2 C), 112.6, 111.7, 108.2, 71.7; IR (film) $v_{\max } 3054,2362,1496,1458,1257,1122$, 
1087, 1042, 998, 836, $748 \mathrm{~cm}^{-1}$; HRMS (ESI), $m / z$ calcd for $\mathrm{C}_{13} \mathrm{H}_{11} \mathrm{BrO}_{3} \mathrm{Na}$ : 316.9789; found: 316.9780 .

6-Bromo-7-methoxybenzo[d][1,3] dioxole-5-carbaldehyde (12). A mixture of 3-(benzyloxy)-5bromo-1, 2-diol (11) (2.5 g, $8.47 \mathrm{mmol}), \mathrm{K}_{2} \mathrm{CO}_{3}(2.53 \mathrm{~g}, 18.6 \mathrm{mmol})$ and bromochloromethane $(0.55 \mathrm{~mL}, 8.47 \mathrm{mmol})$ in DMF $(20 \mathrm{~mL})$ was heated at $100{ }^{\circ} \mathrm{C}$ for $2 \mathrm{~h}$. A further portion of bromochloromethane $(0.55 \mathrm{~mL}, 8.47 \mathrm{mmol})$ was added and heating was continued for $1 \mathrm{~h}$. The solution was allowed to cool to $25^{\circ} \mathrm{C}$, diluted with water $(200 \mathrm{~mL})$ and extracted with diethyl ether $(2 \times 100 \mathrm{~mL})$. The combined organic layers were washed with water and saturated aqueous $\mathrm{NaCl}$. The organic layer was dried $\left(\mathrm{Na}_{2} \mathrm{SO}_{4}\right)$, concentrated, and the residue was purified by flash chromatography (silica, 10\% EtOAc/hexane) to afford $12(1.62 \mathrm{~g}, 96 \%)$ as a colorless solid: ${ }^{1} \mathrm{H}$ $\operatorname{NMR}\left(\mathrm{CDCl}_{3}, 500 \mathrm{MHz}\right) \delta$ 7.45-7.30 (m, 5H), $6.75(\mathrm{~d}, 1 \mathrm{H}, J=1.7 \mathrm{~Hz}), 6.69$ (d, 1H, $\left.J=1.7 \mathrm{~Hz}\right)$, $5.98(\mathrm{~s}, 2 \mathrm{H}), 5.15$ (s, 2H); ${ }^{13} \mathrm{C} \mathrm{NMR}\left(\mathrm{CDCl}_{3}, 125 \mathrm{MHz}\right) \delta 149.7,143.1,136.2,135.3,128.6$, 128.3, 127.6, 113.2, 106.5, 101.8, 71.8; IR (film) $v_{\max } 3054,2362,1492,1296,1024,1050,967$, $832 \mathrm{~cm}^{-1}$; HRMS (ESI), m/z calcd for $\mathrm{C}_{14} \mathrm{H}_{11} \mathrm{BrO}_{3} \mathrm{Na}$ : 328.9789; found: 328.9778 .

$((1 S, 2 S, 3 S)-4-(4-(b e n z y l o x y) b e n z o[d][1,3]$ dioxol-6-yl)-1-(5-bromo-4-methoxybenzo[d][1,3] dioxol-6-yl)-2,3-dimethylbutoxy)(tert-butyl)dimethylsilane (17): A solution of 15 (0.25g, 0.56 $\mathrm{mmol})$ in THF $(2 \mathrm{~mL})$ was cooled to $0{ }^{\circ} \mathrm{C}$, and 9 -BBN in THF $(0.5 \mathrm{M}, 1.42 \mathrm{~mL}, 0.81 \mathrm{mmol})$ was added drop wise by syringe over $25 \mathrm{~min}$ at this temperature. The reaction mixture was allowed to warm to $25^{\circ} \mathrm{C}$ over $4 \mathrm{~h}$, and was stirred at this temperature for $6 \mathrm{~h}$ then transferred drop wise to a flask containing cooled solution of aryl bromide $12(0.38 \mathrm{~g}, 1.23 \mathrm{mmol}), \mathrm{Pd}\left(\mathrm{PPh}_{3}\right)_{4}(0.039 \mathrm{~g}, 0.06$ mmol) and aqueous $\mathrm{NaOH}(0.074 \mathrm{~g}, 1.84 \mathrm{mmol})$ in THF $(2 \mathrm{~mL})$ at $0{ }^{\circ} \mathrm{C}$. The reaction mixture was heated at $70{ }^{\circ} \mathrm{C}$ for $22 \mathrm{~h}$ and cooled to $25^{\circ} \mathrm{C}$ then volatiles were removed, and the residue was diluted with $\mathrm{CH}_{2} \mathrm{Cl}_{2}(20 \mathrm{~mL})$, passed through bed of celite pad, filtrate was washed with water and saturated aqueous $\mathrm{NaCl}$. The organic layer was dried $\left(\mathrm{Na}_{2} \mathrm{SO}_{4}\right)$, concentrated, and the residue was purified by flash chromatography (5-10\% EtOAc/hexane) to afford $17(0.29 \mathrm{~g}, 77 \%)$ as a syrup: ${ }^{1} \mathrm{H} \mathrm{NMR}\left(\mathrm{CDCl}_{3}, 500 \mathrm{MHz}\right) \delta 7.44(\mathrm{~d}, 2 \mathrm{H}, J=7.3 \mathrm{~Hz}), 7.38(2 \mathrm{~d}, 2 \mathrm{H}, J=7.7 \mathrm{~Hz})$, $7.32(2 \mathrm{~d}, 1 \mathrm{H}, J=7.3 \mathrm{~Hz}), 6.79(\mathrm{~s}, 1 \mathrm{H}), 6.42(\operatorname{app~s}, 1 \mathrm{H}), 6.40(\mathrm{~d}, 1 \mathrm{H}, J=0.9 \mathrm{~Hz}), 5.96(\mathrm{ABq}$, $2 \mathrm{H}, J=1.4 \mathrm{~Hz}, \Delta v=20.5 \mathrm{~Hz}), 5.94(\mathrm{~s}, 2 \mathrm{H}), 5.17(\mathrm{~s}, 2 \mathrm{H}), 5.09(\mathrm{~d}, 1 \mathrm{H}, J=7.7 \mathrm{~Hz}), 4.04(\mathrm{~s}, 3 \mathrm{H})$, $2.96(\mathrm{dd}, 1 \mathrm{H}, J=13.2,3.0 \mathrm{~Hz}), 2.30-2.15(\mathrm{~m}, 1 \mathrm{H}), 2.08(\mathrm{dd}, 1 \mathrm{H}, J=13.1,11.8 \mathrm{~Hz}), 1.80$ (m, $1 \mathrm{H}), 0.90(\mathrm{~s}, 9 \mathrm{H}), 0.75(\mathrm{~d}, 3 \mathrm{H}, J=6.8 \mathrm{~Hz}), 0.72(\mathrm{~d}, 3 \mathrm{H}, J=7.2 \mathrm{~Hz}), 0.12(\mathrm{~s}, 3 \mathrm{H})-0.26(\mathrm{~s}, 3 \mathrm{H})$; 
${ }^{13} \mathrm{C} \mathrm{NMR}\left(\mathrm{CDCl}_{3}, 125 \mathrm{MHz}\right) \delta 148.8$ (2 C), 142.3, 139.5, 138.8, 137.0, 136.7, 136.3, 133.5, 128.5, 128.0, 127.6, 110.3, 107.7, 103.5, 102.9, 101.6, 101.1, 76.0, 71.4, 60.1, 46.3, 37.2, 34.0, 26.0, 18.2, 18.1, $10.5-4.5,-4.7$; IR (film) $v_{\max } 2952,2860,1632,1604,1508,1472,1405,1374$, 1255, 1083, 1046, 960, 934, 854, 837, 777, 736, $\mathrm{cm}^{-1}$; HRMS (ESI), $\mathrm{m} / z$ calcd for $\mathrm{C}_{34} \mathrm{H}_{43} \mathrm{BrO}_{7} \mathrm{SiNa}$ : 693.1859; found: 693.1849.

1, 4-Diaryl butane 18. A solution of diaryl butane 17 (0.2 g, $0.29 \mathrm{mmol})$ and 5\% Pd-C (15 mg) in EtOAc $(5 \mathrm{~mL})$ was vigorously stirred at room temperature for $6 \mathrm{~h}$ under hydrogen atmosphere (balloon). The reaction mixture was filtered, filtrate was concentrated, and the residue was purified by flash chromatography (20\% EtOAc/hexane) to afford $18(0.17 \mathrm{~g}, 98 \%)$ as a syrup: ${ }^{1} \mathrm{H}$ $\operatorname{NMR}\left(\mathrm{CDCl}_{3}, 500 \mathrm{MHz}\right) \delta 6.78(\mathrm{~s}, 1 \mathrm{H}), 6.35(\mathrm{~d}, 1 \mathrm{H}, J=1.2 \mathrm{~Hz}), 6.33($ app s, 1H), $5.98(\mathrm{ABq}$, $2 \mathrm{H}, J=1.4 \mathrm{~Hz}, \Delta v=12.2 \mathrm{~Hz}), 5.93(\mathrm{~s}, 2 \mathrm{H}), 5.09$ (d, 1H, $J=7.8 \mathrm{~Hz}), 4.75$ (br s, 1H), 4.03 (s, $3 \mathrm{H}), 2.93(\mathrm{dd}, 1 \mathrm{H}, J=13.2,3.2 \mathrm{~Hz}), 2.35-2.15(\mathrm{~m}, 1 \mathrm{H}), 2.08$ (dd, 1H, $J=13.2,11.8 \mathrm{~Hz}), 1.90-$ $1.75(\mathrm{~m}, 1 \mathrm{H}), 0.91(\mathrm{~s}, 9 \mathrm{H}), 0.78(\mathrm{~d}, 3 \mathrm{H}, J=6.9 \mathrm{~Hz}), 0.71(\mathrm{~d}, 3 \mathrm{H}, J=7.2 \mathrm{~Hz}), 0.10(\mathrm{~s}, 3 \mathrm{H})-0.27$ $(\mathrm{s}, 3 \mathrm{H}) ;{ }^{13} \mathrm{C} \mathrm{NMR}\left(\mathrm{CDCl}_{3}, 125 \mathrm{MHz}\right) \delta 148.8,148.6,139.4,138.8,138.7,137.0,136.3,131.9$, $111.3,107.8,102.9,102.7,101.6,101.2,75.9,60.2$, 46.5, 37.1, 34.0, 26.0, 18.3, 18.1, 10.5 -4.5, -4.7; IR (neat) $v_{\max } 2956,2857,1634,1608,1506,1474,1372,1277,1256,1191,1125,1045$, 960, 857, 834, 776, $736 \mathrm{~cm}^{-1}$; HRMS (ESI), $\mathrm{m} / z$ calcd for $\mathrm{C}_{27} \mathrm{H}_{37} \mathrm{BrO}_{7} \mathrm{SiNa}$ : 603.1390; found: 603.1383.

1, 4-Diaryl butane 19. A solution of 1, 4-diaryl butane 18 (0.15 g, $0.25 \mathrm{mmol})$ in dioxane (5 $\mathrm{mL}$ ) was cooled to $15^{\circ} \mathrm{C}$. A solution of $N$-bromosuccinimide (46 mg, $\left.0.26 \mathrm{mmol}\right)$ in dioxane (3 $\mathrm{mL}$ ) was added drop wise by syringe over $1 \mathrm{~h}$ at this temperature. The reaction mixture was stirred for $11 \mathrm{~h}$ between $15^{\circ} \mathrm{C}$ to $19^{\circ} \mathrm{C}$ and was quenched by the addition of saturated aqueous $\mathrm{Na}_{2} \mathrm{~S}_{2} \mathrm{O}_{3}(1 \mathrm{~mL})$. The mixture was concentrated, and the residue was dissolved in EtOAc (25 $\mathrm{mL}$ ) and washed with saturated aqueous $\mathrm{Na}_{2} \mathrm{~S}_{2} \mathrm{O}_{3}$, water, and saturated aqueous $\mathrm{NaCl}$. The organic layer was dried $\left(\mathrm{Na}_{2} \mathrm{SO}_{4}\right)$, concentrated, and the residue was purified by flash chromatography (silica, 15\% EtOAc/hexane) to afford $19(0.15 \mathrm{~g}, 90 \%)$ as a syrup: ${ }^{1} \mathrm{H}$ NMR $\left(\mathrm{CDCl}_{3}, 500 \mathrm{MHz}\right) \delta 6.80(\mathrm{~s}, 1 \mathrm{H}), 6.46(\mathrm{~s}, 1 \mathrm{H}), 5.99(\mathrm{~s}, 2 \mathrm{H}), 5.98(\mathrm{ABq}, 2 \mathrm{H}, J=1.4 \mathrm{~Hz}, \Delta v=$ $13.6 \mathrm{~Hz}), 5.52$ (s, 1H), 5.09 (d, 1H, $J=7.5 \mathrm{~Hz}), 4.04$ (s, 3H), 2.94 (dd, 1H, $J=13.9,4.2 \mathrm{~Hz})$, $2.59(\mathrm{dd}, 1 \mathrm{H}, J=13.9,11.1 \mathrm{~Hz}), 2.30-2.15(\mathrm{~m}, 1 \mathrm{H}), 1.90-1.75(\mathrm{~m}, 1 \mathrm{H}), 0.89(\mathrm{~s}, 9 \mathrm{H}), 0.81$ (d, 3H, 
$J=7.0 \mathrm{~Hz}), 0.79(\mathrm{~d}, 3 \mathrm{H}, J=7.2 \mathrm{~Hz}), 0.10(\mathrm{~s}, 3 \mathrm{H}), 0.27(\mathrm{~s}, 3 \mathrm{H}) ;{ }^{13} \mathrm{C} \mathrm{NMR}\left(\mathrm{CDCl}_{3}, 125 \mathrm{MHz}\right) \delta$ 148.7, 148.2, 139.5, 138.6, 136.3 (2 C), 134.9, 132.4, 107.7, 106.6, 103.0, 102.9, 101.9, 101.6, $76.1,60.1,46.1,37.3,33.7,26.0,18.0,17.7,11.4,-4.5,-4.8$; IR (neat) $v_{\max } 2956,2886,2355$, 1630, 1605, 1476, 1275, 1251, 1188, 1125, 1084, 959, 932, 837, 776, $735 \mathrm{~cm}^{-1}$; HRMS (ESI), $m / z$ calcd for $\mathrm{C}_{27} \mathrm{H}_{36} \mathrm{Br}_{2} \mathrm{O}_{7} \mathrm{SiNa}$ : 681.0495; found: 681.0515 .

((1S, 2S, 3S)-1, 4-bis(5-bromo-4-methoxybenzo[d][1,3]dioxol-6-yl)-2,3-dimethylbutoxy)(tertbutyl)dimethylsilane (20). A mixture of $19(0.1 \mathrm{~g}, 0.15 \mathrm{mmol}), \mathrm{K}_{2} \mathrm{CO}_{3}(25 \mathrm{mg}, 0.22 \mathrm{mmol})$ and dimethyl sulfate $(15 \mu \mathrm{mL}, 0.18 \mathrm{mmol})$ in acetone $(5 \mathrm{~mL})$ was heated at $56{ }^{\circ} \mathrm{C}$ for $2 \mathrm{~h}$. After cooling to room temperature, the mixture was filtered and concentrated. The residue was dissolved in EtOAc $(50 \mathrm{~mL})$, and was washed with water and saturated aqueous $\mathrm{NaCl}$. The organic layer was dried $\left(\mathrm{Na}_{2} \mathrm{SO}_{4}\right)$, concentrated, and the residue was purified by flash chromatography (silica, 15\% EtOAc/hexane) to afford $20(0.096 \mathrm{~g}, 95 \%)$ as colorless solid: ${ }^{1} \mathrm{H}$ NMR $\left(\mathrm{CDCl}_{3}, 500 \mathrm{MHz}\right) \delta 6.80(\mathrm{~s}, 1 \mathrm{H}), 6.53(\mathrm{~s}, 1 \mathrm{H}), 5.98(\mathrm{ABq}, 2 \mathrm{H}, J=1.4 \mathrm{~Hz}, \Delta v=14.6 \mathrm{~Hz})$, 5.95 (s, 2H), $5.10(\mathrm{~d}, 1 \mathrm{H}, J=7.6 \mathrm{~Hz}), 4.03(\mathrm{~s}, 3 \mathrm{H}), 4.02$ (s, 3H), $2.92(\mathrm{dd}, 1 \mathrm{H}, J=13.8,4.2 \mathrm{~Hz})$, $2.67(\mathrm{dd}, 1 \mathrm{H}, J=13.8,11.0 \mathrm{~Hz}), 2.35-2.20(\mathrm{~m}, 1 \mathrm{H}), 1.90-1.80(\mathrm{~m}, 1 \mathrm{H}), 0.89(\mathrm{~s}, 9 \mathrm{H}), 0.82(\mathrm{~d}, 3 \mathrm{H}$, $J=7.0 \mathrm{~Hz}), 0.78(\mathrm{~d}, 3 \mathrm{H}, J=7.2 \mathrm{~Hz}), 0.10(\mathrm{~s}, 3 \mathrm{H}),-0.27(\mathrm{~s}, 3 \mathrm{H}) ;{ }^{13} \mathrm{C} \mathrm{NMR}\left(\mathrm{CDCl}_{3}, 125 \mathrm{MHz}\right) \delta$ 148.8, 148.3, 140.3, 139.5, 138.7, 136.4, 135.8, 135.5, 110.0, 107.7, 104.6, 103.0, 101.6, 101.4, 76.1, 60.1, 60.0, 46.3, 37.3, 33.8, 26.0, 18.1, 17.7, 11.5, -4.5, -4.7; IR (film) $v_{\max } 2956,2884$, 2856, 1606, 1499, 1473, 1447, 1398, 1373, 1277, 1211, 1085, 1049, 1004, 960, 935, 856, 836, $776,674 \mathrm{~cm}^{-1}$; HRMS (ESI), $m / z$ calcd for $\mathrm{C}_{28} \mathrm{H}_{38} \mathrm{Br}_{2} \mathrm{O}_{7} \mathrm{SiNa}$ : 695.0651; found: 695.0687.

Dibenzocyclooctadiene 21. A solution of $20(0.05 \mathrm{~g}, 0.074 \mathrm{mmol})$ in MeTHF (2 mL) was treated with $t$ - $\mathrm{BuLi}(2.3 \mathrm{M}, 0.13 \mathrm{~mL}, 0.29 \mathrm{mmol})$ at $-78{ }^{\circ} \mathrm{C}$ under argon. After $15 \mathrm{~min}$, the pale yellow colored reaction mixture was transferred via cannula to a flask containing $\mathrm{CuCN}$ ( $7.0 \mathrm{mg}$, $0.074 \mathrm{mmol})$ in MeTHF $(2 \mathrm{~mL})$ at $-78{ }^{\circ} \mathrm{C}$ under argon. The heterogeneous mixture was allowed to warm to $-40{ }^{\circ} \mathrm{C}$ over $1 \mathrm{~h}$, and was stirred until homogeneous at this temperature. The resulting solution was treated with a freshly prepared $0.5 \mathrm{M}$ solution of 1,3-dinitrobenzene $(0.59 \mathrm{~mL}, 0.29$ mmol) at $-40{ }^{\circ} \mathrm{C}$ and was allowed to warm to $25^{\circ} \mathrm{C}$. The reaction mixture was stirred at this temperature for $10 \mathrm{~h}$ and quenched by the addition of $10 \% \mathrm{NH}_{4} \mathrm{OH}$ in saturated aqueous $\mathrm{NH}_{4} \mathrm{Cl}$. The resulting two-phase mixture was stirred for $30 \mathrm{~min}$, and then separated; the aqueous phase 
was extracted two times with EtOAc $(20 \mathrm{~mL})$. The combined organic layers were washed with water and saturated aqueous $\mathrm{NaCl}$. The organic layer was dried $\left(\mathrm{Na}_{2} \mathrm{SO}_{4}\right)$, concentrated, and the residue was purified by flash chromatography (5\% EtOAc/hexane) to afford 21 (26 mg, 69\%) as colorless solid: ${ }^{1} \mathrm{H} \mathrm{NMR}\left(\mathrm{CDCl}_{3}, 500 \mathrm{MHz}\right) \delta 6.87(\mathrm{~s}, 1 \mathrm{H}), 6.43(\mathrm{~s}, 1 \mathrm{H}), 5.96$ (app t, 2H, J = 1.2 $\mathrm{Hz}), 5.95(\mathrm{ABq}, 2 \mathrm{H}, J=1.5 \mathrm{~Hz}, \Delta v=27.9 \mathrm{~Hz}), 4.37$ (d, 1H, $J=1.3 \mathrm{~Hz}), 3.84(\mathrm{~s}, 3 \mathrm{H}), 3.82$ (s, $3 \mathrm{H}), 2.12(\mathrm{dd}, 1 \mathrm{H}, J=13.3,9.2 \mathrm{~Hz}), 1.93(\mathrm{~d}, 1 \mathrm{H}, J=13.3 \mathrm{~Hz}), 1.90-1.80(\mathrm{~m}, 2 \mathrm{H}), 0.97$ (d, 3H, $J$ $=6.9 \mathrm{~Hz}), 0.83(\mathrm{~s}, 9 \mathrm{H}), 0.65(\mathrm{~d}, 3 \mathrm{H}, J=6.9 \mathrm{~Hz}),-0.14(\mathrm{~s}, 3 \mathrm{H}),-0.19(\mathrm{~s}, 3 \mathrm{H}) ;{ }^{13} \mathrm{C} \mathrm{NMR}\left(\mathrm{CDCl}_{3}\right.$, $125 \mathrm{MHz}) \delta 148.9,147.8,140.8,140.3,138.2,136.2,134.8,134.0,120.0,119.5,103.2,102.6$, 100.7, 100.6, 73.6, 59.6, 59.4, 44.4, 39.3, 34.7, 25.9, 22.0, 18.1, 7.8, -4.8, -5.2; IR (film) $v_{\max }$ 2954, 2885, 1632, 1607, 1506, 1474, 1400, 1277, 1252, 1134, 1087, 1046, 960, 936, 857, 776, $736,678 \mathrm{~cm}^{-1}$; HRMS (ESI), $m / z$ calcd for $\mathrm{C}_{28} \mathrm{H}_{38} \mathrm{O}_{7} \mathrm{SiNa}$ : 537.2285; found: 537.2279.

Dibenzocyclooctadiene 22. A solution of $n-\mathrm{Bu}_{4} \mathrm{NF}(56 \mathrm{mg}, 0.21 \mathrm{mmol})$ in THF $(2 \mathrm{~mL})$ was added to a solution of $21(25 \mathrm{mg}, 0.048 \mathrm{mmol})$ in THF $(2 \mathrm{~mL})$ at $55{ }^{\circ} \mathrm{C}$ and the reaction mixture was stirred for $12 \mathrm{~h}$ at this temperature. The volatiles were removed and the residue was dissolved in EtOAc $(10 \mathrm{~mL})$, and was washed with water and saturated aqueous $\mathrm{NaCl}$. The organic layer was dried $\left(\mathrm{Na}_{2} \mathrm{SO}_{4}\right)$, concentrated, and the residue was purified by flash chromatography (30\% EtOAc/hexane) to afford 22 (19 mg, 89\%) as colorless solid: ${ }^{1} \mathrm{H}$ NMR $\left(\mathrm{CDCl}_{3}, 500 \mathrm{MHz}\right) \delta 6.91(\mathrm{~s}, 1 \mathrm{H}), 6.45(\mathrm{~s}, 1 \mathrm{H}), 5.98(\mathrm{ABq}, 2 \mathrm{H}, J=1.5 \mathrm{~Hz}, \Delta v=6.6 \mathrm{~Hz}), 5.94$ $(\mathrm{ABq}, 2 \mathrm{H}, J=1.4 \mathrm{~Hz}, \Delta v=3.0 \mathrm{~Hz}), 4.52(\mathrm{~d}, 1 \mathrm{H}, J=1.4 \mathrm{~Hz}), 3.87(\mathrm{~s}, 3 \mathrm{H}), 3.85(\mathrm{~s}, 3 \mathrm{H}), 2.12$ (dd, $1 \mathrm{H}, J=13.4,9.5 \mathrm{~Hz}), 2.05-1.80(\mathrm{~m}, 3 \mathrm{H}), 1.54$ (br s, 1H), 1.00 (d, 3H, J=7.2 Hz), 0.70 (d, $3 \mathrm{H}, J=7.1 \mathrm{~Hz}) ;{ }^{13} \mathrm{C} \mathrm{NMR}\left(\mathrm{CDCl}_{3}, 125 \mathrm{MHz}\right) \delta 149.1,148.3,140.7,140.6,138.2,135.5,135.2$, 134.3, 120.4, 119.3, 103.0, 102.1, 100.9, 100.8, 73.3, 59.7, 59.6, 42.5, 39.2, 34.6, 21.9, 14.1, 7.8; IR (film) $v_{\max } 3470,2956,2882,1632,1608,1472,1405,1277,1252,1132,1046,960,934$, 832, 736, $674 \mathrm{~cm}^{-1}$; HRMS (ESI), $m / z$ calcd for $\mathrm{C}_{22} \mathrm{H}_{24} \mathrm{O}_{7} \mathrm{Na}$ : 423.1420; found: 423.1427 .

Interiotherin A (1). A solution of $22(12.0 \mathrm{mg}, 0.03 \mathrm{mmol}), \mathrm{PPh}_{3}(32.0 \mathrm{mg}, 0.12 \mathrm{mmol})$ and benzoic acid $(14.0 \mathrm{mg}, 0.12 \mathrm{mmol})$ in THF $(1 \mathrm{~mL})$ were vigorously stirred at $0{ }^{\circ} \mathrm{C}$ for $10 \mathrm{~min}$. Diisopropylazodicarboxylate $(24.0 \mu \mathrm{mL}, 0.13 \mathrm{mmol})$ was added drop wise over a period of 5 min, and the solution was allowed to warm to $25^{\circ} \mathrm{C}$, stirred for $10 \mathrm{~h}$ at this temperature. The reaction mixture was diluted with EtOAc $(10 \mathrm{~mL})$ and was washed with water and saturated 
aqueous $\mathrm{NaCl}$. The organic layer was dried $\left(\mathrm{Na}_{2} \mathrm{SO}_{4}\right)$, concentrated, and the residue was purified by flash chromatography (5\% EtOAc/hexane) to afford interiotherin A (1) $(9.0 \mathrm{mg}, 62 \%)$ as a solid: ${ }^{1} \mathrm{H}$ NMR $\left(\mathrm{CDCl}_{3}, 500 \mathrm{MHz}\right) \delta 7.64(\mathrm{~d}, 2 \mathrm{H}, J=7.3 \mathrm{~Hz}), 7.50-7.40(\mathrm{~m}, 1 \mathrm{H}), 7.35-7.30$ (m, 2H), $6.69(\mathrm{~s}, 1 \mathrm{H}), 6.49(\mathrm{~s}, 1 \mathrm{H}), 5.98(\mathrm{ABq}, 2 \mathrm{H}, J=1.5 \mathrm{~Hz}, \Delta v=21.8 \mathrm{~Hz}), 5.88(\mathrm{ABq}, 2 \mathrm{H}, J=$ $1.5 \mathrm{~Hz}, \Delta v=10.2 \mathrm{~Hz}$ ), 5.86 (overlapping d, $1 \mathrm{H}, J=6.0 \mathrm{~Hz}$ ), 3.79 (s, 3H), 3.58 (s, 3H), 2.26 (d, $2 \mathrm{H}, J=6.5 \mathrm{~Hz}), 2.15-2.00(\mathrm{~m}, 2 \mathrm{H}), 1.02(\mathrm{~d}, 3 \mathrm{H}, J=7.0 \mathrm{~Hz}), 0.86(\mathrm{~d}, 3 \mathrm{H}, J=6.9 \mathrm{~Hz}) ;{ }^{13} \mathrm{C} \mathrm{NMR}$ $\left(\mathrm{CDCl}_{3}, 125 \mathrm{MHz}\right) \delta 165.3,148.8,141.6,141.2,136.8,134.2,132.6,131.1,129.7$ (2C), 127.9 (2C), 122.4 121.5, 106.3, 102.5, 101.2, 100.6, 81.4, 59.7, 59.0, 37.6, 36.6 (2C), 19.3, 14.1; IR (film) $v_{\max } 2924,2358,1713,1616,1475,1450,1372,1269,1211,1101,1070,1049,936,711$ $\mathrm{cm}^{-1}$; HRMS (ESI), $m / z$ calcd for $\mathrm{C}_{29} \mathrm{H}_{28} \mathrm{O}_{8} \mathrm{Na}$ : 527.1682; found: 527.1641 .

Angeloylgomisin R (2). Following the procedure for the preparation of interiotherin A, a solution of diisopropylazodicarboxylate $(16 \mu \mathrm{mL}, 0.08 \mathrm{mmol})$ was added to a solution of $22(8.0$ $\mathrm{mg}, 0.02 \mathrm{mmol}), \mathrm{PPh}_{3}(21.0 \mathrm{mg}, 0.08 \mathrm{mmol})$ and angelic acid $(8.0 \mathrm{mg}, 0.08 \mathrm{mmol})$ in THF (1 $\mathrm{mL})$ to afford angeloylgomisin $\mathrm{R}(2)(5.0 \mathrm{mg}, 51 \%)$ as a syrup. ${ }^{1} \mathrm{H} \mathrm{NMR}\left(\mathrm{CDCl}_{3}, 500 \mathrm{MHz}\right) \delta$ $6.70(\mathrm{~s}, 1 \mathrm{H}), 6.41(\mathrm{~s}, 1 \mathrm{H}), 5.98(\mathrm{ABq}, 2 \mathrm{H}, J=1.5 \mathrm{~Hz}, \Delta v=15 \mathrm{~Hz}), 5.95-5.90(\mathrm{~m}, 3 \mathrm{H}), 5.68(\mathrm{~d}$, $1 \mathrm{H}, J=8.5 \mathrm{~Hz}), 3.83(\mathrm{~s}, 3 \mathrm{H}), 3.79(\mathrm{~s}, 3 \mathrm{H}), 2.45-2.25(\mathrm{~m}, 3 \mathrm{H}), 2.20-1.95(\mathrm{~m}, 1 \mathrm{H}), 1.85$ (dq, $3 \mathrm{H}, J$ $=7.2,1.4 \mathrm{~Hz}), 1.59$ (q, $3 \mathrm{H}, J=1.4 \mathrm{~Hz}), 0.94(\mathrm{~d}, 3 \mathrm{H}, J=7.0 \mathrm{~Hz}), 0.84(\mathrm{~d}, 3 \mathrm{H}, J=6.8 \mathrm{~Hz}) ; \mathrm{IR}$ (film) $v_{\max } 2924,2358,1710,1638,1611,1450,1374,1250,1211,1100,1070,1049,936 \mathrm{~cm}^{-1}$; HRMS (ESI), $m / z$ calcd for $\mathrm{C}_{27} \mathrm{H}_{30} \mathrm{O}_{8} \mathrm{Na}$ : 505.1838; found: 505.1827.

$((1 S, 2 S, 3 S)$-1-(5-bromo-4-methoxybenzo[d][1,3]dioxol-6-yl)-4-(4-methoxybenzo[d][1,3] di oxol-6-yl)-2,3-dimethylbutoxy)(tert-butyl)dimethylsilane (25). Following the procedure for the preparation of 1, 4-diaryl butane 17, 9-BBN in THF $(0.5 \mathrm{M}, 0.65 \mathrm{~mL}, 0.32 \mathrm{mmol})$ was added to a solution of $15(0.1 \mathrm{~g}, 0.22 \mathrm{mmol})$ in THF $(1 \mathrm{~mL})$, transferred to a flask containing 6-bromo-4methoxy benzo[d][1,3]dioxole (24) $(0.1 \mathrm{~g}, 0.44 \mathrm{mmol}), \mathrm{Pd}\left(\mathrm{PPh}_{3}\right)_{4}(0.016 \mathrm{~g}, 0.014 \mathrm{mmol})$ and aqueous $\mathrm{NaOH}(0.03 \mathrm{~g}, 0.73 \mathrm{mmol})$ in THF $(2 \mathrm{~mL})$ at $0{ }^{\circ} \mathrm{C}$ and the reaction mixture was heated at $70{ }^{\circ} \mathrm{C}$ for $16 \mathrm{~h}$ to afford $25(0.096 \mathrm{~g}, 72 \%)$ as a syrup: ${ }^{1} \mathrm{H} \mathrm{NMR}\left(\mathrm{CDCl}_{3}, 500 \mathrm{MHz}\right) \delta 6.78(\mathrm{~s}$, 1H), 6.39 (d, 1H, $J=1.1 \mathrm{~Hz}), 6.37$ (app s, 1H), $5.98(\mathrm{ABq}, 2 \mathrm{H}, J=1.4 \mathrm{~Hz}, \Delta v=16.5 \mathrm{~Hz}), 5.93$ (s, 2H), 5.10 (d, 1H, J= 7.8 Hz), 4.04 (s, 3H), 3.90 (s, 3H), 2.99 (dd, 1H, $J=13.2,3.2 \mathrm{~Hz}), 2.30-$ $2.15(\mathrm{~m}, 1 \mathrm{H}), 2.11(\mathrm{dd}, 1 \mathrm{H}, J=13.1,11.7 \mathrm{~Hz}), 1.80(\mathrm{qd}, 1 \mathrm{H}, J=7.4,1.7 \mathrm{~Hz}), 0.91$ (s, 9H), 0.79 
$(\mathrm{d}, 3 \mathrm{H}, J=6.8 \mathrm{~Hz}), 0.73(\mathrm{~d}, 3 \mathrm{H}, J=7.2 \mathrm{~Hz}), 0.13(\mathrm{~s}, 3 \mathrm{H})-0.25(\mathrm{~s}, 3 \mathrm{H}) ;{ }^{13} \mathrm{C} \mathrm{NMR}\left(\mathrm{CDCl}_{3}, 125\right.$ MHz) $\delta$ 148.7, 148.6, 143.3, 139.4, 138.7, 136.8, 136.3, 133.0, 107.9, 107.7, 103.1, 102.8, 101.5, 101.1, 75.9, 60.1, 56.4, 46.3, 37.2, 33.9, 25.9, 18.3, 18.0, 10.5, -4.5, -4.8; IR (film) $v_{\max } 2954$, 2884, 2856, 1632, 1608, 1508, 1472, 1400, 1374, 1277, 1252, 1132, 1087, 1046, 960, 934, 857, 837, 776, 736, $678 \mathrm{~cm}^{-1}$; HRMS (ESI), $\mathrm{m} / z$ calcd for $\mathrm{C}_{28} \mathrm{H}_{39} \mathrm{BrO}_{7} \mathrm{SiNa}$ : 617.1546; found: 617.1541 .

((1S, 2S, 3S)-1,4-bis(5-bromo-4-methoxybenzo[d][1,3]dioxol-6-yl)-2,3-dimethylbutoxy)(tertbutyl)dimethylsilane (20). A solution of $25(0.09 \mathrm{~g}, 0.15 \mathrm{mmol})$ in $\mathrm{CHCl}_{3}(2 \mathrm{~mL})$ was cooled to $15^{\circ} \mathrm{C}$ to $19{ }^{\circ} \mathrm{C}$. A solution of $N$-bromosuccinimide $(0.03 \mathrm{~g}, 0.16 \mathrm{mmol})$ in $\mathrm{CHCl}_{3}(6 \mathrm{~mL})$ was added drop wise by syringe over $1 \mathrm{~h}$ at this temperature. The reaction mixture was stirred for $6 \mathrm{~h}$ at $25{ }^{\circ} \mathrm{C}$ and was quenched by the addition of saturated aqueous $\mathrm{Na}_{2} \mathrm{~S}_{2} \mathrm{O}_{3}(1 \mathrm{~mL})$. The mixture was concentrated, and the residue was dissolved in EtOAc $(25 \mathrm{~mL})$ and washed with saturated aqueous $\mathrm{Na}_{2} \mathrm{~S}_{2} \mathrm{O}_{3}$, water, and saturated aqueous $\mathrm{NaCl}$. The organic layer was dried $\left(\mathrm{Na}_{2} \mathrm{SO}_{4}\right)$, concentrated, and the residue was purified by flash chromatography (silica, 15\% EtOAc/hexane) to afford $20(0.083 \mathrm{~g}, 82 \%)$ as colorless solid. 


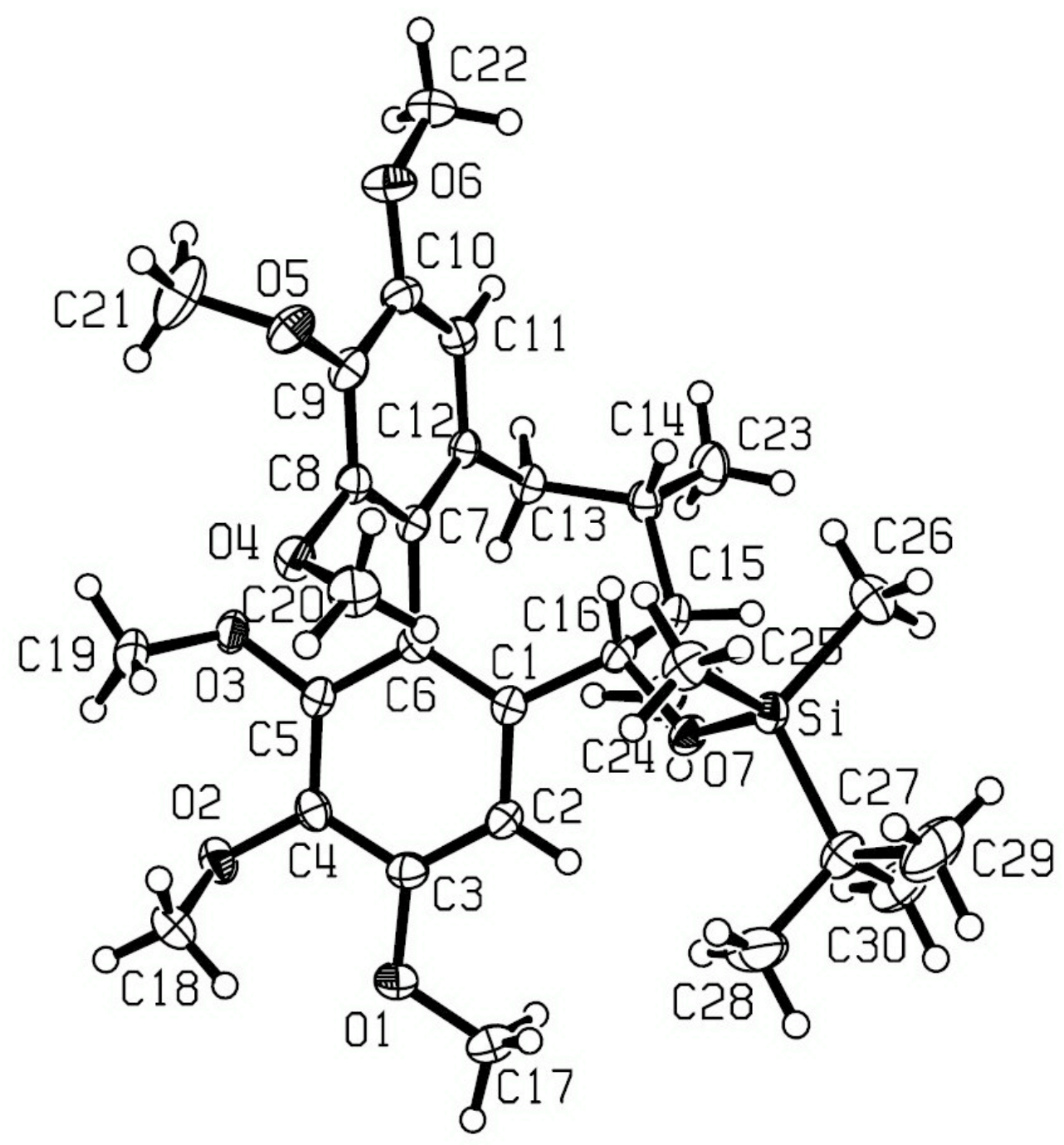

\section{X-ray Crystallographic Data for dibenzocyclooctadiene 23 (Coleman 1300)}

The data collection crystal was a clear, colorless block cut from the end of a rectangular block. Examination of the diffraction pattern on a Nonius Kappa CCD diffractometer indicated a triclinic crystal system. All work was done at 150 K using an Oxford Cryosystems Cryostream Cooler. The data collection strategy was set up to measure a hemisphere of reciprocal space with a redundancy factor of 3.1, which means that $90 \%$ of the reflections were measured at least 3.1 times. Phi and omega scans with a frame width of $1.0^{\circ}$ were used for data collection. Data integration was done with Dingo (1), and scaling and merging of the data was done with Scalepack(1). Merging the data and averaging the symmetry equivalent reflections resulted in a Rint value of 0.037 . 
The structure was solved by the direct methods procedure in SHELXS-97 (2) in $\overline{\mathrm{H}}$. Full-matrix least-squares refinements based on $\mathrm{F}^{2}$ were performed in SHELXL-97 (3), as incorporated in the WingX package (4). There is a solvent molecule of hexane present on a crystallographic inversion center.

For the methyl groups, the hydrogen atoms were added at calculated positions using a riding model with $\mathrm{U}(\mathrm{H})=1.5$ * Ueq(bonded carbon atom). The torsion angle, which defines the orientation of the methyl group about the C-C, O-C or Si-c bond, was refined. The remaining hydrogen atoms were included in the model at calculated positions using a riding model with $\mathrm{U}(\mathrm{H})=1.2$ * Ueq(attached atom). The final refinement cycle was based on 7749 intensities and 384 variables and resulted in agreement factors of $R 1(F)=0.062$ and $\mathrm{wR} 2\left(\mathrm{~F}^{2}\right)=0.114$. For the subset of data with $\mathrm{I}>2 * \mathrm{sigma}$ (I), the R1 (F) value is 0.041 for 5838 reflections. The final difference electron density map contains maximum and minimum peak heights of 0.48 and -0.32 e/ 3 . Neutral atom scattering factors were used and include terms for anomalous dispersion (5).

References

(1) DENZO: Otwinowski, Z. \& Minor, W., Methods in Enzymology, Vol 276: Macromolecular Crystallography, part A, 307-326, (1997), Carter, Jr., C. W. \& Sweet, R. M., Eds., Academic Press.

(2) SHELXS-97: Sheldrick, G. M., Universitat Gottingen, Germany, 1997 .

(3) SHELXL-97: Sheldrick, G. M., Universitat Gottingen, Germany, 1997 .

(4) WingX-Version 1.64.05: Farrugia, L. J., J. Appl. Cryst., (1999), $32,837-838$.

(5) International Tables for Crystallography (1992). Volume C. Dordrecht: Kluwer Academic Publishers. 
Table 1. Crystallographic details for Dibenzocyclooctadiene 23 (Coleman 1300)

\begin{tabular}{|c|c|c|c|}
\hline Empirical formula & \multicolumn{3}{|c|}{$\mathrm{C} 30 \mathrm{H} 46 \mathrm{O} 7 \mathrm{Si}+_{-}$hexane } \\
\hline Formula weight & \multicolumn{3}{|l|}{589.84} \\
\hline Temperature & \multicolumn{3}{|l|}{$150(2) \mathrm{K}$} \\
\hline & \multicolumn{2}{|l|}{ Wavelength } & $0.71073 \AA$ \\
\hline Crystal system & \multicolumn{3}{|l|}{ triclinic } \\
\hline Space group & \multicolumn{3}{|l|}{$\overline{\mathrm{P}}$} \\
\hline \multirow[t]{3}{*}{ Unit cell dimensions } & $a=10.691(1) \AA$ & $\alpha=84.330(5)^{\circ}$ & \\
\hline & $\mathrm{b}=11.526(1) \AA$ & $\beta=75.379(5)^{\circ}$ & \\
\hline & $\mathrm{c}=14.901(2) \AA$ & $\gamma=72.421(5)^{\circ}$ & \\
\hline Volume & \multicolumn{3}{|l|}{$1693.4(3) \AA^{3}$} \\
\hline $\mathrm{Z}$ & \multicolumn{3}{|l|}{2} \\
\hline Density (calculated) & \multicolumn{3}{|l|}{$1.157 \mathrm{Mg} / \mathrm{m}^{3}$} \\
\hline Absorption coefficient & \multicolumn{3}{|l|}{$0.112 \mathrm{~mm}^{-1}$} \\
\hline $\mathrm{F}(000)$ & \multicolumn{3}{|l|}{642} \\
\hline Crystal size & \multicolumn{3}{|c|}{$0.15 \times 0.25 \times 0.30 \mathrm{~mm}^{3}$} \\
\hline Theta range for data collection & \multicolumn{3}{|l|}{2.34 to $27.49^{\circ}$} \\
\hline Index ranges & \multicolumn{3}{|c|}{$-13<=\mathrm{h}<=13,-14<=\mathrm{k}<=14,-19<=\mathrm{l}<=19$} \\
\hline Reflections collected & \multicolumn{3}{|l|}{45191} \\
\hline Independent reflections & \multicolumn{3}{|c|}{$7749[\mathrm{R}(\mathrm{int})=0.037]$} \\
\hline Completeness to theta $=27.49^{\circ}$ & \multicolumn{3}{|l|}{$99.7 \%$} \\
\hline Refinement method & \multicolumn{3}{|c|}{ Full-matrix least-squares on $\mathrm{F}^{2}$} \\
\hline Data / restraints / parameters & \multicolumn{3}{|l|}{$7749 / 0 / 384$} \\
\hline Goodness-of-fit on $\mathrm{F}^{2}$ & \multicolumn{3}{|l|}{1.046} \\
\hline Final $R$ indices $[I>2 \operatorname{sigma}(I)]$ & \multicolumn{3}{|c|}{$\mathrm{R} 1=0.0411, \mathrm{wR} 2=0.1048$} \\
\hline $\mathrm{R}$ indices (all data) & \multicolumn{3}{|c|}{$\mathrm{R} 1=0.0624, \mathrm{wR} 2=0.1144$} \\
\hline Largest diff. peak and hole & \multicolumn{3}{|c|}{0.480 and $-0.316 \mathrm{e} / \AA^{3}$} \\
\hline
\end{tabular}

Table 2. Atomic coordinates $\left(\times 10^{4}\right)$ and equivalent isotropic displacement parameters $\left(\AA^{2} \times 10^{3}\right)$

for Dibenzocycooctadiene 23 (Coleman 1300). U (eq) is defined as one third of the trace of the orthogonalized $U^{i j}$ tensor.

\begin{tabular}{ccccc}
\hline & $\mathrm{x}$ & $\mathrm{y}$ & $\mathrm{z}$ & $\mathrm{U}(\mathrm{eq})$ \\
\hline $\mathrm{C}(1)$ & $8245(1)$ & $-3276(1)$ & $2521(1)$ & $20(1)$
\end{tabular}




\begin{tabular}{|c|c|c|c|c|}
\hline$C(2)$ & $7552(1)$ & $-3495(1)$ & 1904(1) & $23(1)$ \\
\hline$C(3)$ & 7911(1) & $-4626(1)$ & $1520(1)$ & $24(1)$ \\
\hline$C(4)$ & 8961(1) & $-5575(1)$ & $1753(1)$ & $23(1)$ \\
\hline$C(5)$ & $9628(1)$ & $-5366(1)$ & $2384(1)$ & $21(1)$ \\
\hline$C(6)$ & 9301(1) & $-4206(1)$ & $2757(1)$ & $19(1)$ \\
\hline$C(7)$ & $10169(1)$ & $-4002(1)$ & $3335(1)$ & 19(1) \\
\hline$C(8)$ & $11543(1)$ & $-4147(1)$ & 2922(1) & $20(1)$ \\
\hline$C(9)$ & $12408(1)$ & $-3958(1)$ & $3404(1)$ & $23(1)$ \\
\hline$C(10)$ & $11920(1)$ & $-3651(1)$ & $4340(1)$ & $23(1)$ \\
\hline $\mathrm{C}(11)$ & $10575(1)$ & $-3515(1)$ & $4762(1)$ & $22(1)$ \\
\hline $\mathrm{C}(12)$ & $9691(1)$ & $-3681(1)$ & $4266(1)$ & $19(1)$ \\
\hline $\mathrm{C}(13)$ & $8229(1)$ & $-3467(1)$ & $4762(1)$ & $22(1)$ \\
\hline$C(14)$ & $7380(1)$ & $-2126(1)$ & 4693(1) & $23(1)$ \\
\hline $\mathrm{C}(15)$ & $6755(1)$ & $-1805(1)$ & $3841(1)$ & $23(1)$ \\
\hline$C(16)$ & 7821(1) & $-2004(1)$ & $2906(1)$ & $21(1)$ \\
\hline $\mathrm{C}(17)$ & $6153(2)$ & $-3996(2)$ & $702(1)$ & $41(1)$ \\
\hline $\mathrm{C}(18)$ & $9966(2)$ & $-6789(2)$ & $433(1)$ & $37(1)$ \\
\hline$C(19)$ & $11653(2)$ & $-7066(2)$ & 2097(1) & $37(1)$ \\
\hline$C(20)$ & $12129(2)$ & $-3514(2)$ & $1372(1)$ & $35(1)$ \\
\hline$C(21)$ & $14639(2)$ & $-5205(2)$ & $3102(1)$ & $49(1)$ \\
\hline$C(22)$ & $12443(2)$ & $-3327(2)$ & $5741(1)$ & $34(1)$ \\
\hline$C(23)$ & $6296(2)$ & $-1769(2)$ & $5589(1)$ & $37(1)$ \\
\hline$C(24)$ & $5622(2)$ & $-2369(2)$ & $3872(1)$ & $33(1)$ \\
\hline$C(25)$ & $9465(2)$ & $-217(2)$ & $1324(1)$ & $31(1)$ \\
\hline$C(26)$ & $7256(2)$ & $1165(1)$ & $2837(1)$ & $33(1)$ \\
\hline$C(27)$ & $6539(2)$ & $787(1)$ & $1032(1)$ & $28(1)$ \\
\hline$C(28)$ & $6898(2)$ & $-63(2)$ & $217(1)$ & $45(1)$ \\
\hline$C(29)$ & $6747(2)$ & 2012(2) & $651(2)$ & $56(1)$ \\
\hline$C(30)$ & $5059(2)$ & $960(2)$ & $1528(1)$ & $45(1)$ \\
\hline $\mathrm{C}(31)$ & $678(2)$ & $9875(2)$ & $4665(1)$ & $51(1)$ \\
\hline$C(32)$ & $737(2)$ & $9704(2)$ & $3678(2)$ & $63(1)$ \\
\hline $\mathrm{C}(33)$ & $2147(2)$ & $9417(2)$ & $3038(2)$ & $57(1)$ \\
\hline $\mathrm{O}(1)$ & $7284(1)$ & $-4916(1)$ & $908(1)$ & $34(1)$ \\
\hline $\mathrm{O}(2)$ & $9282(1)$ & $-6723(1)$ & $1398(1)$ & $30(1)$ \\
\hline $\mathrm{O}(3)$ & $10556(1)$ & $-6285(1)$ & $2730(1)$ & $26(1)$ \\
\hline $\mathrm{O}(4)$ & 12061(1) & $-4510(1)$ & 2010(1) & $24(1)$ \\
\hline
\end{tabular}




\begin{tabular}{lrrrr}
$\mathrm{O}(5)$ & $13736(1)$ & $-4051(1)$ & $2960(1)$ & $28(1)$ \\
$\mathrm{O}(6)$ & $12860(1)$ & $-3519(1)$ & $4768(1)$ & $32(1)$ \\
$\mathrm{O}(7)$ & $7253(1)$ & $-1160(1)$ & $2236(1)$ & $24(1)$ \\
$\mathrm{Si}$ & $7633(1)$ & $114(1)$ & $1872(1)$ & $20(1)$ \\
\hline
\end{tabular}

Table 3. Bond lengths $[\AA]$ and angles $\left[{ }^{\circ}\right]$ for Dibenzocyclooctadiene 23 (Coleman 1300).

\begin{tabular}{ll}
\hline $\mathrm{C}(1)-\mathrm{C}(6)$ & $1.3937(19)$ \\
$\mathrm{C}(1)-\mathrm{C}(2)$ & $1.3993(19)$ \\
$\mathrm{C}(1)-\mathrm{C}(16)$ & $1.5226(19)$ \\
$\mathrm{C}(2)-\mathrm{C}(3)$ & $1.382(2)$ \\
$\mathrm{C}(2)-\mathrm{H}(2)$ & 0.9500 \\
$\mathrm{C}(3)-\mathrm{O}(1)$ & $1.3772(17)$ \\
$\mathrm{C}(3)-\mathrm{C}(4)$ & $1.400(2)$ \\
$\mathrm{C}(4)-\mathrm{O}(2)$ & $1.3849(16)$ \\
$\mathrm{C}(4)-\mathrm{C}(5)$ & $1.3918(19)$ \\
$\mathrm{C}(5)-\mathrm{O}(3)$ & $1.3745(16)$ \\
$\mathrm{C}(5)-\mathrm{C}(6)$ & $1.4088(19)$ \\
$\mathrm{C}(6)-\mathrm{C}(7)$ & $1.4945(18)$ \\
$\mathrm{C}(7)-\mathrm{C}(12)$ & $1.3966(18)$ \\
$\mathrm{C}(7)-\mathrm{C}(8)$ & $1.4073(19)$ \\
$\mathrm{C}(8)-\mathrm{C}(9)$ & $1.3814(19)$ \\
$\mathrm{C}(8)-\mathrm{O}(4)$ & $1.3860(16)$ \\
$\mathrm{C}(9)-\mathrm{O}(5)$ & $1.3830(16)$ \\
$\mathrm{C}(9)-\mathrm{C}(10)$ & $1.400(2)$ \\
$\mathrm{C}(10)-\mathrm{O}(6)$ & $1.3690(16)$ \\
$\mathrm{C}(10)-\mathrm{C}(11)$ & $1.383(2)$ \\
$\mathrm{C}(11)-\mathrm{C}(12)$ & $1.4021(19)$ \\
$\mathrm{C}(11)-\mathrm{H}(11)$ & 0.9500 \\
$\mathrm{C}(12)-\mathrm{C}(13)$ & $1.5070(18)$ \\
$\mathrm{C}(13)-\mathrm{C}(14)$ & $1.5472(19)$ \\
$\mathrm{C}(13)-\mathrm{H}(13 \mathrm{~A})$ & 0.9900 \\
$\mathrm{C}(13)-\mathrm{H}(13 \mathrm{~B})$ & 0.9900 \\
$\mathrm{C}(14)-\mathrm{C}(23)$ & $1.528(2)$ \\
$\mathrm{C}(14)-\mathrm{C}(15)$ & $1.5430(19)$ \\
&
\end{tabular}




\begin{tabular}{|c|c|}
\hline $\mathrm{C}(14)-\mathrm{H}(14)$ & 1.0000 \\
\hline$C(15)-C(24)$ & $1.527(2)$ \\
\hline$C(15)-C(16)$ & $1.5481(19)$ \\
\hline $\mathrm{C}(15)-\mathrm{H}(15)$ & 1.0000 \\
\hline $\mathrm{C}(16)-\mathrm{O}(7)$ & $1.4378(16)$ \\
\hline $\mathrm{C}(16)-\mathrm{H}(16)$ & 1.0000 \\
\hline $\mathrm{C}(17)-\mathrm{O}(1)$ & $1.4246(19)$ \\
\hline $\mathrm{C}(17)-\mathrm{H}(17 \mathrm{~A})$ & 0.9800 \\
\hline $\mathrm{C}(17)-\mathrm{H}(17 \mathrm{~B})$ & 0.9800 \\
\hline $\mathrm{C}(17)-\mathrm{H}(17 \mathrm{C})$ & 0.9800 \\
\hline $\mathrm{C}(18)-\mathrm{O}(2)$ & $1.4367(19)$ \\
\hline $\mathrm{C}(18)-\mathrm{H}(18 \mathrm{~A})$ & 0.9800 \\
\hline $\mathrm{C}(18)-\mathrm{H}(18 \mathrm{~B})$ & 0.9800 \\
\hline $\mathrm{C}(18)-\mathrm{H}(18 \mathrm{C})$ & 0.9800 \\
\hline $\mathrm{C}(19)-\mathrm{O}(3)$ & $1.4311(18)$ \\
\hline $\mathrm{C}(19)-\mathrm{H}(19 \mathrm{~A})$ & 0.9800 \\
\hline $\mathrm{C}(19)-\mathrm{H}(19 \mathrm{~B})$ & 0.9800 \\
\hline $\mathrm{C}(19)-\mathrm{H}(19 \mathrm{C})$ & 0.9800 \\
\hline $\mathrm{C}(20)-\mathrm{O}(4)$ & $1.4232(18)$ \\
\hline $\mathrm{C}(20)-\mathrm{H}(20 \mathrm{~A})$ & 0.9800 \\
\hline $\mathrm{C}(20)-\mathrm{H}(20 \mathrm{~B})$ & 0.9800 \\
\hline $\mathrm{C}(20)-\mathrm{H}(20 \mathrm{C})$ & 0.9800 \\
\hline $\mathrm{C}(21)-\mathrm{O}(5)$ & $1.421(2)$ \\
\hline $\mathrm{C}(21)-\mathrm{H}(21 \mathrm{~A})$ & 0.9800 \\
\hline $\mathrm{C}(21)-\mathrm{H}(21 \mathrm{~B})$ & 0.9800 \\
\hline $\mathrm{C}(21)-\mathrm{H}(21 \mathrm{C})$ & 0.9800 \\
\hline $\mathrm{C}(22)-\mathrm{O}(6)$ & $1.4245(18)$ \\
\hline $\mathrm{C}(22)-\mathrm{H}(22 \mathrm{~A})$ & 0.9800 \\
\hline $\mathrm{C}(22)-\mathrm{H}(22 \mathrm{~B})$ & 0.9800 \\
\hline $\mathrm{C}(22)-\mathrm{H}(22 \mathrm{C})$ & 0.9800 \\
\hline $\mathrm{C}(23)-\mathrm{H}(23 \mathrm{~A})$ & 0.9800 \\
\hline $\mathrm{C}(23)-\mathrm{H}(23 \mathrm{~B})$ & 0.9800 \\
\hline $\mathrm{C}(23)-\mathrm{H}(23 \mathrm{C})$ & 0.9800 \\
\hline $\mathrm{C}(24)-\mathrm{H}(24 \mathrm{~A})$ & 0.9800 \\
\hline $\mathrm{C}(24)-\mathrm{H}(24 \mathrm{~B})$ & 0.9800 \\
\hline $\mathrm{C}(24)-\mathrm{H}(24 \mathrm{C})$ & 0.9800 \\
\hline
\end{tabular}




\begin{tabular}{|c|c|}
\hline $\mathrm{C}(25)-\mathrm{Si}$ & $1.8583(15)$ \\
\hline $\mathrm{C}(25)-\mathrm{H}(25 \mathrm{~A})$ & 0.9800 \\
\hline $\mathrm{C}(25)-\mathrm{H}(25 \mathrm{~B})$ & 0.9800 \\
\hline $\mathrm{C}(25)-\mathrm{H}(25 \mathrm{C})$ & 0.9800 \\
\hline $\mathrm{C}(26)-\mathrm{Si}$ & $1.8584(15)$ \\
\hline $\mathrm{C}(26)-\mathrm{H}(26 \mathrm{~A})$ & 0.9800 \\
\hline $\mathrm{C}(26)-\mathrm{H}(26 \mathrm{~B})$ & 0.9800 \\
\hline $\mathrm{C}(26)-\mathrm{H}(26 \mathrm{C})$ & 0.9800 \\
\hline $\mathrm{C}(27)-\mathrm{C}(29)$ & $1.526(2)$ \\
\hline$C(27)-C(30)$ & $1.529(2)$ \\
\hline $\mathrm{C}(27)-\mathrm{C}(28)$ & $1.535(2)$ \\
\hline $\mathrm{C}(27)-\mathrm{Si}$ & $1.8811(15)$ \\
\hline $\mathrm{C}(28)-\mathrm{H}(28 \mathrm{~A})$ & 0.9800 \\
\hline $\mathrm{C}(28)-\mathrm{H}(28 \mathrm{~B})$ & 0.9800 \\
\hline $\mathrm{C}(28)-\mathrm{H}(28 \mathrm{C})$ & 0.9800 \\
\hline $\mathrm{C}(29)-\mathrm{H}(29 \mathrm{~A})$ & 0.9800 \\
\hline $\mathrm{C}(29)-\mathrm{H}(29 \mathrm{~B})$ & 0.9800 \\
\hline C(29)-H(29C) & 0.9800 \\
\hline $\mathrm{C}(30)-\mathrm{H}(30 \mathrm{~A})$ & 0.9800 \\
\hline $\mathrm{C}(30)-\mathrm{H}(30 \mathrm{~B})$ & 0.9800 \\
\hline $\mathrm{C}(30)-\mathrm{H}(30 \mathrm{C})$ & 0.9800 \\
\hline $\mathrm{C}(31)-\mathrm{C}(32)$ & $1.486(3)$ \\
\hline $\mathrm{C}(31)-\mathrm{C}(31) \# 1$ & $1.501(4)$ \\
\hline $\mathrm{C}(31)-\mathrm{H}(31 \mathrm{~A})$ & 0.9900 \\
\hline $\mathrm{C}(31)-\mathrm{H}(31 \mathrm{~B})$ & 0.9900 \\
\hline $\mathrm{C}(32)-\mathrm{C}(33)$ & $1.525(3)$ \\
\hline $\mathrm{C}(32)-\mathrm{H}(32 \mathrm{~A})$ & 0.9900 \\
\hline $\mathrm{C}(32)-\mathrm{H}(32 \mathrm{~B})$ & 0.9900 \\
\hline $\mathrm{C}(33)-\mathrm{H}(33 \mathrm{~A})$ & 0.9800 \\
\hline $\mathrm{C}(33)-\mathrm{H}(33 \mathrm{~B})$ & 0.9800 \\
\hline $\mathrm{C}(33)-\mathrm{H}(33 \mathrm{C})$ & 0.9800 \\
\hline $\mathrm{O}(7)-\mathrm{Si}$ & $1.6451(10)$ \\
\hline$C(6)-C(1)-C(2)$ & $119.99(12)$ \\
\hline$C(6)-C(1)-C(16)$ & $121.92(12)$ \\
\hline$C(2)-C(1)-C(16)$ & $118.09(12)$ \\
\hline$C(3)-C(2)-C(1)$ & $120.58(13)$ \\
\hline
\end{tabular}




\begin{tabular}{|c|c|}
\hline $\mathrm{C}(3)-\mathrm{C}(2)-\mathrm{H}(2)$ & 119.7 \\
\hline $\mathrm{C}(1)-\mathrm{C}(2)-\mathrm{H}(2)$ & 119.7 \\
\hline $\mathrm{O}(1)-\mathrm{C}(3)-\mathrm{C}(2)$ & $124.27(13)$ \\
\hline $\mathrm{O}(1)-\mathrm{C}(3)-\mathrm{C}(4)$ & $115.33(12)$ \\
\hline $\mathrm{C}(2)-\mathrm{C}(3)-\mathrm{C}(4)$ & $120.39(13)$ \\
\hline $\mathrm{O}(2)-\mathrm{C}(4)-\mathrm{C}(5)$ & $120.57(12)$ \\
\hline $\mathrm{O}(2)-\mathrm{C}(4)-\mathrm{C}(3)$ & $120.35(12)$ \\
\hline$C(5)-C(4)-C(3)$ & $118.99(12)$ \\
\hline $\mathrm{O}(3)-\mathrm{C}(5)-\mathrm{C}(4)$ & $122.31(12)$ \\
\hline $\mathrm{O}(3)-\mathrm{C}(5)-\mathrm{C}(6)$ & $116.38(12)$ \\
\hline$C(4)-C(5)-C(6)$ & $121.12(12)$ \\
\hline$C(1)-C(6)-C(5)$ & $118.86(12)$ \\
\hline $\mathrm{C}(1)-\mathrm{C}(6)-\mathrm{C}(7)$ & $122.61(12)$ \\
\hline$C(5)-C(6)-C(7)$ & $118.37(12)$ \\
\hline $\mathrm{C}(12)-\mathrm{C}(7)-\mathrm{C}(8)$ & $118.13(12)$ \\
\hline $\mathrm{C}(12)-\mathrm{C}(7)-\mathrm{C}(6)$ & $123.51(12)$ \\
\hline $\mathrm{C}(8)-\mathrm{C}(7)-\mathrm{C}(6)$ & $118.36(12)$ \\
\hline $\mathrm{C}(9)-\mathrm{C}(8)-\mathrm{O}(4)$ & $118.40(12)$ \\
\hline $\mathrm{C}(9)-\mathrm{C}(8)-\mathrm{C}(7)$ & $121.96(12)$ \\
\hline $\mathrm{O}(4)-\mathrm{C}(8)-\mathrm{C}(7)$ & $119.63(12)$ \\
\hline $\mathrm{C}(8)-\mathrm{C}(9)-\mathrm{O}(5)$ & $120.11(12)$ \\
\hline C(8)-C(9)-C(10) & $119.28(12)$ \\
\hline $\mathrm{O}(5)-\mathrm{C}(9)-\mathrm{C}(10)$ & $120.61(12)$ \\
\hline $\mathrm{O}(6)-\mathrm{C}(10)-\mathrm{C}(11)$ & $125.21(13)$ \\
\hline $\mathrm{O}(6)-\mathrm{C}(10)-\mathrm{C}(9)$ & $115.11(12)$ \\
\hline $\mathrm{C}(11)-\mathrm{C}(10)-\mathrm{C}(9)$ & $119.68(12)$ \\
\hline $\mathrm{C}(10)-\mathrm{C}(11)-\mathrm{C}(12)$ & $120.95(12)$ \\
\hline $\mathrm{C}(10)-\mathrm{C}(11)-\mathrm{H}(11)$ & 119.5 \\
\hline $\mathrm{C}(12)-\mathrm{C}(11)-\mathrm{H}(11)$ & 119.5 \\
\hline$C(7)-C(12)-C(11)$ & $119.98(12)$ \\
\hline$C(7)-C(12)-C(13)$ & $122.17(12)$ \\
\hline $\mathrm{C}(11)-\mathrm{C}(12)-\mathrm{C}(13)$ & $117.82(12)$ \\
\hline $\mathrm{C}(12)-\mathrm{C}(13)-\mathrm{C}(14)$ & $112.94(11)$ \\
\hline $\mathrm{C}(12)-\mathrm{C}(13)-\mathrm{H}(13 \mathrm{~A})$ & 109.0 \\
\hline $\mathrm{C}(14)-\mathrm{C}(13)-\mathrm{H}(13 \mathrm{~A})$ & 109.0 \\
\hline $\mathrm{C}(12)-\mathrm{C}(13)-\mathrm{H}(13 \mathrm{~B})$ & 109.0 \\
\hline
\end{tabular}




\begin{tabular}{|c|c|}
\hline $\mathrm{C}(14)-\mathrm{C}(13)-\mathrm{H}(13 \mathrm{~B})$ & 109.0 \\
\hline $\mathrm{H}(13 \mathrm{~A})-\mathrm{C}(13)-\mathrm{H}(13 \mathrm{~B})$ & 107.8 \\
\hline $\mathrm{C}(23)-\mathrm{C}(14)-\mathrm{C}(15)$ & $110.83(12)$ \\
\hline$C(23)-C(14)-C(13)$ & $110.30(12)$ \\
\hline$C(15)-C(14)-C(13)$ & $114.94(11)$ \\
\hline $\mathrm{C}(23)-\mathrm{C}(14)-\mathrm{H}(14)$ & 106.8 \\
\hline $\mathrm{C}(15)-\mathrm{C}(14)-\mathrm{H}(14)$ & 106.8 \\
\hline $\mathrm{C}(13)-\mathrm{C}(14)-\mathrm{H}(14)$ & 106.8 \\
\hline$C(24)-C(15)-C(14)$ & $114.27(12)$ \\
\hline$C(24)-C(15)-C(16)$ & $112.55(12)$ \\
\hline$C(14)-C(15)-C(16)$ & $113.28(11)$ \\
\hline $\mathrm{C}(24)-\mathrm{C}(15)-\mathrm{H}(15)$ & 105.2 \\
\hline $\mathrm{C}(14)-\mathrm{C}(15)-\mathrm{H}(15)$ & 105.2 \\
\hline $\mathrm{C}(16)-\mathrm{C}(15)-\mathrm{H}(15)$ & 105.2 \\
\hline $\mathrm{O}(7)-\mathrm{C}(16)-\mathrm{C}(1)$ & $107.68(10)$ \\
\hline $\mathrm{O}(7)-\mathrm{C}(16)-\mathrm{C}(15)$ & $107.44(10)$ \\
\hline $\mathrm{C}(1)-\mathrm{C}(16)-\mathrm{C}(15)$ & $115.33(11)$ \\
\hline $\mathrm{O}(7)-\mathrm{C}(16)-\mathrm{H}(16)$ & 108.7 \\
\hline $\mathrm{C}(1)-\mathrm{C}(16)-\mathrm{H}(16)$ & 108.7 \\
\hline $\mathrm{C}(15)-\mathrm{C}(16)-\mathrm{H}(16)$ & 108.7 \\
\hline $\mathrm{O}(1)-\mathrm{C}(17)-\mathrm{H}(17 \mathrm{~A})$ & 109.5 \\
\hline $\mathrm{O}(1)-\mathrm{C}(17)-\mathrm{H}(17 \mathrm{~B})$ & 109.5 \\
\hline $\mathrm{H}(17 \mathrm{~A})-\mathrm{C}(17)-\mathrm{H}(17 \mathrm{~B})$ & 109.5 \\
\hline $\mathrm{O}(1)-\mathrm{C}(17)-\mathrm{H}(17 \mathrm{C})$ & 109.5 \\
\hline $\mathrm{H}(17 \mathrm{~A})-\mathrm{C}(17)-\mathrm{H}(17 \mathrm{C})$ & 109.5 \\
\hline $\mathrm{H}(17 \mathrm{~B})-\mathrm{C}(17)-\mathrm{H}(17 \mathrm{C})$ & 109.5 \\
\hline $\mathrm{O}(2)-\mathrm{C}(18)-\mathrm{H}(18 \mathrm{~A})$ & 109.5 \\
\hline $\mathrm{O}(2)-\mathrm{C}(18)-\mathrm{H}(18 \mathrm{~B})$ & 109.5 \\
\hline $\mathrm{H}(18 \mathrm{~A})-\mathrm{C}(18)-\mathrm{H}(18 \mathrm{~B})$ & 109.5 \\
\hline $\mathrm{O}(2)-\mathrm{C}(18)-\mathrm{H}(18 \mathrm{C})$ & 109.5 \\
\hline $\mathrm{H}(18 \mathrm{~A})-\mathrm{C}(18)-\mathrm{H}(18 \mathrm{C})$ & 109.5 \\
\hline $\mathrm{H}(18 \mathrm{~B})-\mathrm{C}(18)-\mathrm{H}(18 \mathrm{C})$ & 109.5 \\
\hline $\mathrm{O}(3)-\mathrm{C}(19)-\mathrm{H}(19 \mathrm{~A})$ & 109.5 \\
\hline $\mathrm{O}(3)-\mathrm{C}(19)-\mathrm{H}(19 \mathrm{~B})$ & 109.5 \\
\hline H(19A)-C(19)-H(19B) & 109.5 \\
\hline $\mathrm{O}(3)-\mathrm{C}(19)-\mathrm{H}(19 \mathrm{C})$ & 109.5 \\
\hline
\end{tabular}




\begin{tabular}{|c|c|}
\hline $\mathrm{H}(19 \mathrm{~A})-\mathrm{C}(19)-\mathrm{H}(19 \mathrm{C})$ & 109.5 \\
\hline $\mathrm{H}(19 \mathrm{~B})-\mathrm{C}(19)-\mathrm{H}(19 \mathrm{C})$ & 109.5 \\
\hline $\mathrm{O}(4)-\mathrm{C}(20)-\mathrm{H}(20 \mathrm{~A})$ & 109.5 \\
\hline $\mathrm{O}(4)-\mathrm{C}(20)-\mathrm{H}(20 \mathrm{~B})$ & 109.5 \\
\hline $\mathrm{H}(20 \mathrm{~A})-\mathrm{C}(20)-\mathrm{H}(20 \mathrm{~B})$ & 109.5 \\
\hline $\mathrm{O}(4)-\mathrm{C}(20)-\mathrm{H}(20 \mathrm{C})$ & 109.5 \\
\hline $\mathrm{H}(20 \mathrm{~A})-\mathrm{C}(20)-\mathrm{H}(20 \mathrm{C})$ & 109.5 \\
\hline $\mathrm{H}(20 \mathrm{~B})-\mathrm{C}(20)-\mathrm{H}(20 \mathrm{C})$ & 109.5 \\
\hline $\mathrm{O}(5)-\mathrm{C}(21)-\mathrm{H}(21 \mathrm{~A})$ & 109.5 \\
\hline $\mathrm{O}(5)-\mathrm{C}(21)-\mathrm{H}(21 \mathrm{~B})$ & 109.5 \\
\hline $\mathrm{H}(21 \mathrm{~A})-\mathrm{C}(21)-\mathrm{H}(21 \mathrm{~B})$ & 109.5 \\
\hline $\mathrm{O}(5)-\mathrm{C}(21)-\mathrm{H}(21 \mathrm{C})$ & 109.5 \\
\hline $\mathrm{H}(21 \mathrm{~A})-\mathrm{C}(21)-\mathrm{H}(21 \mathrm{C})$ & 109.5 \\
\hline $\mathrm{H}(21 \mathrm{~B})-\mathrm{C}(21)-\mathrm{H}(21 \mathrm{C})$ & 109.5 \\
\hline $\mathrm{O}(6)-\mathrm{C}(22)-\mathrm{H}(22 \mathrm{~A})$ & 109.5 \\
\hline $\mathrm{O}(6)-\mathrm{C}(22)-\mathrm{H}(22 \mathrm{~B})$ & 109.5 \\
\hline $\mathrm{H}(22 \mathrm{~A})-\mathrm{C}(22)-\mathrm{H}(22 \mathrm{~B})$ & 109.5 \\
\hline $\mathrm{O}(6)-\mathrm{C}(22)-\mathrm{H}(22 \mathrm{C})$ & 109.5 \\
\hline $\mathrm{H}(22 \mathrm{~A})-\mathrm{C}(22)-\mathrm{H}(22 \mathrm{C})$ & 109.5 \\
\hline $\mathrm{H}(22 \mathrm{~B})-\mathrm{C}(22)-\mathrm{H}(22 \mathrm{C})$ & 109.5 \\
\hline $\mathrm{C}(14)-\mathrm{C}(23)-\mathrm{H}(23 \mathrm{~A})$ & 109.5 \\
\hline $\mathrm{C}(14)-\mathrm{C}(23)-\mathrm{H}(23 \mathrm{~B})$ & 109.5 \\
\hline $\mathrm{H}(23 \mathrm{~A})-\mathrm{C}(23)-\mathrm{H}(23 \mathrm{~B})$ & 109.5 \\
\hline $\mathrm{C}(14)-\mathrm{C}(23)-\mathrm{H}(23 \mathrm{C})$ & 109.5 \\
\hline $\mathrm{H}(23 \mathrm{~A})-\mathrm{C}(23)-\mathrm{H}(23 \mathrm{C})$ & 109.5 \\
\hline $\mathrm{H}(23 \mathrm{~B})-\mathrm{C}(23)-\mathrm{H}(23 \mathrm{C})$ & 109.5 \\
\hline $\mathrm{C}(15)-\mathrm{C}(24)-\mathrm{H}(24 \mathrm{~A})$ & 109.5 \\
\hline $\mathrm{C}(15)-\mathrm{C}(24)-\mathrm{H}(24 \mathrm{~B})$ & 109.5 \\
\hline $\mathrm{H}(24 \mathrm{~A})-\mathrm{C}(24)-\mathrm{H}(24 \mathrm{~B})$ & 109.5 \\
\hline $\mathrm{C}(15)-\mathrm{C}(24)-\mathrm{H}(24 \mathrm{C})$ & 109.5 \\
\hline $\mathrm{H}(24 \mathrm{~A})-\mathrm{C}(24)-\mathrm{H}(24 \mathrm{C})$ & 109.5 \\
\hline $\mathrm{H}(24 \mathrm{~B})-\mathrm{C}(24)-\mathrm{H}(24 \mathrm{C})$ & 109.5 \\
\hline $\mathrm{Si}-\mathrm{C}(25)-\mathrm{H}(25 \mathrm{~A})$ & 109.5 \\
\hline $\mathrm{Si}-\mathrm{C}(25)-\mathrm{H}(25 \mathrm{~B})$ & 109.5 \\
\hline $\mathrm{H}(25 \mathrm{~A})-\mathrm{C}(25)-\mathrm{H}(25 \mathrm{~B})$ & 109.5 \\
\hline $\mathrm{Si}-\mathrm{C}(25)-\mathrm{H}(25 \mathrm{C})$ & 109.5 \\
\hline
\end{tabular}




\begin{tabular}{|c|c|}
\hline $\mathrm{H}(25 \mathrm{~A})-\mathrm{C}(25)-\mathrm{H}(25 \mathrm{C})$ & 109.5 \\
\hline $\mathrm{H}(25 \mathrm{~B})-\mathrm{C}(25)-\mathrm{H}(25 \mathrm{C})$ & 109.5 \\
\hline $\mathrm{Si}-\mathrm{C}(26)-\mathrm{H}(26 \mathrm{~A})$ & 109.5 \\
\hline $\mathrm{Si}-\mathrm{C}(26)-\mathrm{H}(26 \mathrm{~B})$ & 109.5 \\
\hline $\mathrm{H}(26 \mathrm{~A})-\mathrm{C}(26)-\mathrm{H}(26 \mathrm{~B})$ & 109.5 \\
\hline $\mathrm{Si}-\mathrm{C}(26)-\mathrm{H}(26 \mathrm{C})$ & 109.5 \\
\hline $\mathrm{H}(26 \mathrm{~A})-\mathrm{C}(26)-\mathrm{H}(26 \mathrm{C})$ & 109.5 \\
\hline $\mathrm{H}(26 \mathrm{~B})-\mathrm{C}(26)-\mathrm{H}(26 \mathrm{C})$ & 109.5 \\
\hline $\mathrm{C}(29)-\mathrm{C}(27)-\mathrm{C}(30)$ & $109.81(15)$ \\
\hline $\mathrm{C}(29)-\mathrm{C}(27)-\mathrm{C}(28)$ & $108.63(15)$ \\
\hline $\mathrm{C}(30)-\mathrm{C}(27)-\mathrm{C}(28)$ & $108.57(14)$ \\
\hline $\mathrm{C}(29)-\mathrm{C}(27)-\mathrm{Si}$ & $109.93(11)$ \\
\hline $\mathrm{C}(30)-\mathrm{C}(27)-\mathrm{Si}$ & $109.33(11)$ \\
\hline $\mathrm{C}(28)-\mathrm{C}(27)-\mathrm{Si}$ & $110.54(11)$ \\
\hline $\mathrm{C}(27)-\mathrm{C}(28)-\mathrm{H}(28 \mathrm{~A})$ & 109.5 \\
\hline $\mathrm{C}(27)-\mathrm{C}(28)-\mathrm{H}(28 \mathrm{~B})$ & 109.5 \\
\hline $\mathrm{H}(28 \mathrm{~A})-\mathrm{C}(28)-\mathrm{H}(28 \mathrm{~B})$ & 109.5 \\
\hline $\mathrm{C}(27)-\mathrm{C}(28)-\mathrm{H}(28 \mathrm{C})$ & 109.5 \\
\hline $\mathrm{H}(28 \mathrm{~A})-\mathrm{C}(28)-\mathrm{H}(28 \mathrm{C})$ & 109.5 \\
\hline $\mathrm{H}(28 \mathrm{~B})-\mathrm{C}(28)-\mathrm{H}(28 \mathrm{C})$ & 109.5 \\
\hline $\mathrm{C}(27)-\mathrm{C}(29)-\mathrm{H}(29 \mathrm{~A})$ & 109.5 \\
\hline $\mathrm{C}(27)-\mathrm{C}(29)-\mathrm{H}(29 \mathrm{~B})$ & 109.5 \\
\hline $\mathrm{H}(29 \mathrm{~A})-\mathrm{C}(29)-\mathrm{H}(29 \mathrm{~B})$ & 109.5 \\
\hline $\mathrm{C}(27)-\mathrm{C}(29)-\mathrm{H}(29 \mathrm{C})$ & 109.5 \\
\hline H(29A)-C(29)-H(29C) & 109.5 \\
\hline $\mathrm{H}(29 \mathrm{~B})-\mathrm{C}(29)-\mathrm{H}(29 \mathrm{C})$ & 109.5 \\
\hline $\mathrm{C}(27)-\mathrm{C}(30)-\mathrm{H}(30 \mathrm{~A})$ & 109.5 \\
\hline $\mathrm{C}(27)-\mathrm{C}(30)-\mathrm{H}(30 \mathrm{~B})$ & 109.5 \\
\hline $\mathrm{H}(30 \mathrm{~A})-\mathrm{C}(30)-\mathrm{H}(30 \mathrm{~B})$ & 109.5 \\
\hline $\mathrm{C}(27)-\mathrm{C}(30)-\mathrm{H}(30 \mathrm{C})$ & 109.5 \\
\hline $\mathrm{H}(30 \mathrm{~A})-\mathrm{C}(30)-\mathrm{H}(30 \mathrm{C})$ & 109.5 \\
\hline $\mathrm{H}(30 \mathrm{~B})-\mathrm{C}(30)-\mathrm{H}(30 \mathrm{C})$ & 109.5 \\
\hline $\mathrm{C}(32)-\mathrm{C}(31)-\mathrm{C}(31) \# 1$ & $117.5(2)$ \\
\hline $\mathrm{C}(32)-\mathrm{C}(31)-\mathrm{H}(31 \mathrm{~A})$ & 107.9 \\
\hline $\mathrm{C}(31) \# 1-\mathrm{C}(31)-\mathrm{H}(31 \mathrm{~A})$ & 107.9 \\
\hline $\mathrm{C}(32)-\mathrm{C}(31)-\mathrm{H}(31 \mathrm{~B})$ & 107.9 \\
\hline
\end{tabular}




$\begin{array}{ll}\mathrm{C}(31) \text { \#1-C(31)-H(31B) } & 107.9 \\ \mathrm{H}(31 \mathrm{~A})-\mathrm{C}(31)-\mathrm{H}(31 \mathrm{~B}) & 107.2 \\ \mathrm{C}(31)-\mathrm{C}(32)-\mathrm{C}(33) & 115.01(18) \\ \mathrm{C}(31)-\mathrm{C}(32)-\mathrm{H}(32 \mathrm{~A}) & 108.5 \\ \mathrm{C}(33)-\mathrm{C}(32)-\mathrm{H}(32 \mathrm{~A}) & 108.5 \\ \mathrm{C}(31)-\mathrm{C}(32)-\mathrm{H}(32 \mathrm{~B}) & 108.5 \\ \mathrm{C}(33)-\mathrm{C}(32)-\mathrm{H}(32 \mathrm{~B}) & 108.5 \\ \mathrm{H}(32 \mathrm{~A})-\mathrm{C}(32)-\mathrm{H}(32 \mathrm{~B}) & 107.5 \\ \mathrm{C}(32)-\mathrm{C}(33)-\mathrm{H}(33 \mathrm{~A}) & 109.5 \\ \mathrm{C}(32)-\mathrm{C}(33)-\mathrm{H}(33 \mathrm{~B}) & 109.5 \\ \mathrm{H}(33 \mathrm{~A})-\mathrm{C}(33)-\mathrm{H}(33 \mathrm{~B}) & 109.5 \\ \mathrm{C}(32)-\mathrm{C}(33)-\mathrm{H}(33 \mathrm{C}) & 109.5 \\ \mathrm{H}(33 \mathrm{~A})-\mathrm{C}(33)-\mathrm{H}(33 \mathrm{C}) & 109.5 \\ \mathrm{H}(33 \mathrm{~B})-\mathrm{C}(33)-\mathrm{H}(33 \mathrm{C}) & 109.5 \\ \mathrm{C}(3)-\mathrm{O}(1)-\mathrm{C}(17) & 116.90(12) \\ \mathrm{C}(4)-\mathrm{O}(2)-\mathrm{C}(18) & 113.10(11) \\ \mathrm{C}(5)-\mathrm{O}(3)-\mathrm{C}(19) & 119.06(11) \\ \mathrm{C}(8)-\mathrm{O}(4)-\mathrm{C}(20) & 112.75(11) \\ \mathrm{C}(9)-\mathrm{O}(5)-\mathrm{C}(21) & 113.09(11) \\ \mathrm{C}(10)-\mathrm{O}(6)-\mathrm{C}(22) & 117.13(11) \\ \mathrm{C}(16)-\mathrm{O}(7)-\mathrm{Si} & 125.02(8) \\ \mathrm{O}(7)-\mathrm{Si}-\mathrm{C}(25) & 110.10(6) \\ \mathrm{O}(7)-\mathrm{Si}-\mathrm{C}(26) & 112.15(6) \\ \mathrm{C}(25)-\mathrm{Si}-\mathrm{C}(26) & 107.17(7) \\ \mathrm{O}(7)-\mathrm{Si}-\mathrm{C}(27) & 103.63(6) \\ \mathrm{C}(25)-\mathrm{Si}-\mathrm{C}(27) & 112.96(7) \\ \mathrm{C}(26)-\mathrm{Si}-\mathrm{C}(27) & 110.91(7) \\ & \end{array}$

Symmetry transformations used to generate equivalent atoms:

$\# 1-x,-y+2,-z+1$

Table 4. Anisotropic displacement parameters $\left(\AA^{2} \times 10^{3}\right)$ for Dibenzocyclooctadiene 23 (Coleman 1300). The anisotropic displacement factor exponent takes the form: $-2 \pi^{2}\left[\mathrm{~h}^{2} \mathrm{a}^{* 2} \mathrm{U}^{11}+\ldots+2 \mathrm{hk} \mathrm{a}^{*} \mathrm{~b}^{*} \mathrm{U}^{12}\right]$

$\begin{array}{llllll}\mathrm{U}^{11} & \mathrm{U}^{22} & \mathrm{U}^{33} & \mathrm{U}^{23} & \mathrm{U}^{13} & \mathrm{U}^{12}\end{array}$




\begin{tabular}{|c|c|c|c|c|c|c|}
\hline $\mathrm{C}(1)$ & $18(1)$ & $20(1)$ & $19(1)$ & $1(1)$ & $-2(1)$ & $-6(1)$ \\
\hline$C(2)$ & $20(1)$ & $23(1)$ & $26(1)$ & $1(1)$ & $-7(1)$ & $-5(1)$ \\
\hline $\mathrm{C}(3)$ & $22(1)$ & $29(1)$ & $23(1)$ & $-1(1)$ & $-7(1)$ & $-9(1)$ \\
\hline $\mathrm{C}(4)$ & $23(1)$ & $20(1)$ & $24(1)$ & $-2(1)$ & $-2(1)$ & $-8(1)$ \\
\hline$C(5)$ & $19(1)$ & $20(1)$ & $20(1)$ & $3(1)$ & $-2(1)$ & $-4(1)$ \\
\hline$C(6)$ & $17(1)$ & $22(1)$ & $17(1)$ & $0(1)$ & $-1(1)$ & $-6(1)$ \\
\hline$C(7)$ & $19(1)$ & $17(1)$ & $20(1)$ & $2(1)$ & $-5(1)$ & $-4(1)$ \\
\hline $\mathrm{C}(8)$ & $21(1)$ & $20(1)$ & $18(1)$ & $1(1)$ & $-3(1)$ & $-3(1)$ \\
\hline $\mathrm{C}(9)$ & $17(1)$ & $24(1)$ & $25(1)$ & $4(1)$ & $-3(1)$ & $-6(1)$ \\
\hline$C(10)$ & $25(1)$ & $23(1)$ & $25(1)$ & $4(1)$ & $-10(1)$ & $-9(1)$ \\
\hline $\mathrm{C}(11)$ & $25(1)$ & $21(1)$ & $18(1)$ & $2(1)$ & $-5(1)$ & $-6(1)$ \\
\hline$C(12)$ & $20(1)$ & $16(1)$ & $20(1)$ & $2(1)$ & $-4(1)$ & $-4(1)$ \\
\hline $\mathrm{C}(13)$ & $20(1)$ & $22(1)$ & $21(1)$ & $1(1)$ & $-2(1)$ & $-5(1)$ \\
\hline$C(14)$ & $23(1)$ & $21(1)$ & $21(1)$ & $-2(1)$ & $-1(1)$ & $-4(1)$ \\
\hline$C(15)$ & $20(1)$ & $20(1)$ & $25(1)$ & $0(1)$ & $-3(1)$ & $-1(1)$ \\
\hline$C(16)$ & $20(1)$ & $19(1)$ & $23(1)$ & $3(1)$ & $-7(1)$ & $-4(1)$ \\
\hline $\mathrm{C}(17)$ & $36(1)$ & $45(1)$ & $48(1)$ & $-8(1)$ & $-27(1)$ & $-4(1)$ \\
\hline$C(18)$ & $42(1)$ & $35(1)$ & $32(1)$ & $-14(1)$ & $-9(1)$ & $-5(1)$ \\
\hline $\mathrm{C}(19)$ & $31(1)$ & $32(1)$ & $37(1)$ & $-9(1)$ & $-9(1)$ & $9(1)$ \\
\hline$C(20)$ & $44(1)$ & $38(1)$ & $21(1)$ & $5(1)$ & $-5(1)$ & $-11(1)$ \\
\hline$C(21)$ & $22(1)$ & $49(1)$ & $61(1)$ & $16(1)$ & $-2(1)$ & $0(1)$ \\
\hline$C(22)$ & $37(1)$ & $40(1)$ & $29(1)$ & $-3(1)$ & $-15(1)$ & $-12(1)$ \\
\hline$C(23)$ & $34(1)$ & $36(1)$ & $26(1)$ & $-3(1)$ & $2(1)$ & $3(1)$ \\
\hline$C(24)$ & $20(1)$ & $42(1)$ & $35(1)$ & $1(1)$ & $-4(1)$ & $-7(1)$ \\
\hline$C(25)$ & $25(1)$ & $37(1)$ & $27(1)$ & $1(1)$ & $-4(1)$ & $-7(1)$ \\
\hline$C(26)$ & $34(1)$ & $31(1)$ & $33(1)$ & $-7(1)$ & $-2(1)$ & $-10(1)$ \\
\hline$C(27)$ & $24(1)$ & $29(1)$ & $26(1)$ & $6(1)$ & $-6(1)$ & $-5(1)$ \\
\hline$C(28)$ & $36(1)$ & $68(1)$ & $31(1)$ & $-6(1)$ & $-14(1)$ & $-7(1)$ \\
\hline$C(29)$ & $62(1)$ & $44(1)$ & $70(1)$ & $32(1)$ & $-36(1)$ & $-20(1)$ \\
\hline$C(30)$ & $23(1)$ & $64(1)$ & $39(1)$ & $4(1)$ & $-8(1)$ & $-2(1)$ \\
\hline$C(31)$ & $51(1)$ & $43(1)$ & $68(1)$ & $5(1)$ & $-28(1)$ & $-15(1)$ \\
\hline$C(32)$ & $52(1)$ & $66(1)$ & $74(2)$ & $6(1)$ & $-30(1)$ & $-11(1)$ \\
\hline$C(33)$ & $49(1)$ & $52(1)$ & $72(2)$ & $6(1)$ & $-19(1)$ & $-16(1)$ \\
\hline $\mathrm{O}(1)$ & $31(1)$ & $35(1)$ & $41(1)$ & $-10(1)$ & $-19(1)$ & $-6(1)$ \\
\hline $\mathrm{O}(2)$ & $33(1)$ & $21(1)$ & $34(1)$ & $-6(1)$ & $-6(1)$ & $-8(1)$ \\
\hline $\mathrm{O}(3)$ & $26(1)$ & $21(1)$ & $25(1)$ & $-2(1)$ & $-6(1)$ & $2(1)$ \\
\hline
\end{tabular}




\begin{tabular}{lllllll}
$\mathrm{O}(4)$ & $23(1)$ & $27(1)$ & $18(1)$ & $0(1)$ & $0(1)$ & $-4(1)$ \\
$\mathrm{O}(5)$ & $17(1)$ & $35(1)$ & $30(1)$ & $7(1)$ & $-4(1)$ & $-8(1)$ \\
$\mathrm{O}(6)$ & $28(1)$ & $46(1)$ & $27(1)$ & $0(1)$ & $-10(1)$ & $-17(1)$ \\
$\mathrm{O}(7)$ & $26(1)$ & $20(1)$ & $26(1)$ & $5(1)$ & $-11(1)$ & $-6(1)$ \\
$\mathrm{Si}$ & $20(1)$ & $19(1)$ & $20(1)$ & $0(1)$ & $-3(1)$ & $-4(1)$ \\
\hline
\end{tabular}

Table 5. Calculated hydrogen coordinates $\left(\mathrm{x} 10^{4}\right)$ and isotropic displacement parameters $\left(\AA^{2} \times 10^{3}\right)$ for Dibenzocyclooctadiene 23 (Coleman 1300).

\begin{tabular}{|c|c|c|c|c|}
\hline & $\mathrm{x}$ & $\mathrm{y}$ & $\mathrm{z}$ & $\mathrm{U}(\mathrm{eq})$ \\
\hline $\mathrm{H}(2)$ & 6829 & -2860 & 1749 & 27 \\
\hline $\mathrm{H}(11)$ & 10246 & -3307 & 5397 & 26 \\
\hline $\mathrm{H}(13 \mathrm{~A})$ & 8172 & -3708 & 5424 & 26 \\
\hline $\mathrm{H}(13 \mathrm{~B})$ & 7841 & -3992 & 4495 & 26 \\
\hline $\mathrm{H}(14)$ & 8004 & -1614 & 4638 & 27 \\
\hline $\mathrm{H}(15)$ & 6313 & -906 & 3866 & 28 \\
\hline $\mathrm{H}(16)$ & 8640 & -1814 & 2981 & 25 \\
\hline $\mathrm{H}(17 \mathrm{~A})$ & 5459 & -3758 & 1276 & 62 \\
\hline $\mathrm{H}(17 \mathrm{~B})$ & 5784 & -4311 & 271 & 62 \\
\hline $\mathrm{H}(17 \mathrm{C})$ & 6439 & -3286 & 417 & 62 \\
\hline $\mathrm{H}(18 \mathrm{~A})$ & 9385 & -6222 & 70 & 56 \\
\hline $\mathrm{H}(18 \mathrm{~B})$ & 10173 & -7619 & 223 & 56 \\
\hline $\mathrm{H}(18 \mathrm{C})$ & 10806 & -6570 & 349 & 56 \\
\hline $\mathrm{H}(19 \mathrm{~A})$ & 11361 & -7723 & 1922 & 55 \\
\hline $\mathrm{H}(19 \mathrm{~B})$ & 12409 & -7416 & 2394 & 55 \\
\hline $\mathrm{H}(19 \mathrm{C})$ & 11941 & -6595 & 1540 & 55 \\
\hline $\mathrm{H}(20 \mathrm{~A})$ & 11218 & -2966 & 1405 & 53 \\
\hline $\mathrm{H}(20 \mathrm{~B})$ & 12523 & -3821 & 742 & 53 \\
\hline $\mathrm{H}(20 \mathrm{C})$ & 12692 & -3071 & 1529 & 53 \\
\hline $\mathrm{H}(21 \mathrm{~A})$ & 14561 & -5370 & 3768 & 74 \\
\hline $\mathrm{H}(21 \mathrm{~B})$ & 15566 & -5203 & 2800 & 74 \\
\hline $\mathrm{H}(21 \mathrm{C})$ & 14414 & -5837 & 2834 & 74 \\
\hline $\mathrm{H}(22 \mathrm{~A})$ & 11671 & -2596 & 5870 & 50 \\
\hline
\end{tabular}




\begin{tabular}{|c|c|c|c|c|}
\hline $\mathrm{H}(22 \mathrm{~B})$ & 13190 & -3220 & 5962 & 50 \\
\hline $\mathrm{H}(22 \mathrm{C})$ & 12182 & -4033 & 6061 & 50 \\
\hline $\mathrm{H}(23 \mathrm{~A})$ & 5740 & -930 & 5526 & 55 \\
\hline $\mathrm{H}(23 \mathrm{~B})$ & 6728 & -1829 & 6106 & 55 \\
\hline $\mathrm{H}(23 \mathrm{C})$ & 5726 & -2318 & 5711 & 55 \\
\hline $\mathrm{H}(24 \mathrm{~A})$ & 5959 & -3256 & 3939 & 50 \\
\hline $\mathrm{H}(24 \mathrm{~B})$ & 5310 & -2160 & 3296 & 50 \\
\hline $\mathrm{H}(24 \mathrm{C})$ & 4870 & -2053 & 4401 & 50 \\
\hline $\mathrm{H}(25 \mathrm{~A})$ & 9986 & -641 & 1775 & 46 \\
\hline $\mathrm{H}(25 \mathrm{~B})$ & 9684 & 548 & 1121 & 46 \\
\hline $\mathrm{H}(25 \mathrm{C})$ & 9691 & -732 & 787 & 46 \\
\hline $\mathrm{H}(26 \mathrm{~A})$ & 6279 & 1440 & 3094 & 49 \\
\hline $\mathrm{H}(26 \mathrm{~B})$ & 7584 & 1871 & 2602 & 49 \\
\hline $\mathrm{H}(26 \mathrm{C})$ & 7707 & 740 & 3323 & 49 \\
\hline $\mathrm{H}(28 \mathrm{~A})$ & 6762 & -851 & 452 & 68 \\
\hline $\mathrm{H}(28 \mathrm{~B})$ & 7844 & -180 & -109 & 68 \\
\hline $\mathrm{H}(28 \mathrm{C})$ & 6316 & 299 & -212 & 68 \\
\hline $\mathrm{H}(29 \mathrm{~A})$ & 6189 & 2348 & 203 & 84 \\
\hline $\mathrm{H}(29 \mathrm{~B})$ & 7699 & 1898 & 343 & 84 \\
\hline $\mathrm{H}(29 \mathrm{C})$ & 6484 & 2575 & 1162 & 84 \\
\hline $\mathrm{H}(30 \mathrm{~A})$ & 4811 & 1514 & 2044 & 67 \\
\hline $\mathrm{H}(30 \mathrm{~B})$ & 4931 & 171 & 1767 & 67 \\
\hline $\mathrm{H}(30 \mathrm{C})$ & 4488 & 1306 & 1090 & 67 \\
\hline $\mathrm{H}(31 \mathrm{~A})$ & 1048 & 10559 & 4689 & 62 \\
\hline $\mathrm{H}(31 \mathrm{~B})$ & 1285 & 9134 & 4889 & 62 \\
\hline $\mathrm{H}(32 \mathrm{~A})$ & 160 & 10455 & 3441 & 76 \\
\hline $\mathrm{H}(32 \mathrm{~B})$ & 349 & 9034 & 3649 & 76 \\
\hline $\mathrm{H}(33 \mathrm{~A})$ & 2544 & 10074 & 3060 & 86 \\
\hline $\mathrm{H}(33 \mathrm{~B})$ & 2088 & 9344 & 2402 & 86 \\
\hline $\mathrm{H}(33 \mathrm{C})$ & 2715 & 8649 & 3242 & 86 \\
\hline
\end{tabular}

